
COMMUNICATIONS

ISSN 2056-9890

Received 11 November 2017

Accepted 13 November 2017

Edited by W. T. A. Harrison, University of Aberdeen, Scotland

₹ Correspondence e-mail for synthetic work: Matthias.Wagner@chemie.uni-frankfurt.de.

Keywords: crystal structure; decachlorocyclopentasilanes; inverse-sandwich complex; C-

CCDC references: 1585194; 1585193

Supporting information: this article has supporting information at journals.iucr.org/e $\mathrm{H}$. . Cl contacts.

\section{Decachlorocyclopentasilanes coordinated by pairs of chloride anions, with different cations, but the same solvent molecules}

\author{
Maximilian Moxter, Julian Teichmann, Hans-Wolfram Lerner, Michael Bolte* and \\ Matthias Wagner‡
}

Institut für Anorganische Chemie, J. W. Goethe-Universität Frankfurt, Max-von-Laue-Strasse 7, 60438 Frankfurt/Main, Germany. *Correspondence e-mail: bolte@chemie.uni-frankfurt.de

We have determined the crystal structures of two decachlorocyclopentasilanes, namely bis(tetra- $n$-butylammonium) dichloride decachlorocyclopentasilane dichloromethane disolvate, $2 \mathrm{C}_{16} \mathrm{H}_{36} \mathrm{~N}^{+} \cdot 2 \mathrm{Cl}^{-} \cdot \mathrm{Si}_{5} \mathrm{Cl}_{10} \cdot 2 \mathrm{CH}_{2} \mathrm{Cl}_{2}$, (I), and bis(tetraethylammonium) dichloride decachlorocyclopentasilane dichloromethane disolvate, $2 \mathrm{C}_{8} \mathrm{H}_{20} \mathrm{~N}^{+} \cdot 2 \mathrm{Cl}^{-} \cdot \mathrm{Si}_{5} \mathrm{Cl}_{10} \cdot 2 \mathrm{CH}_{2} \mathrm{Cl}_{2}$, (II), both of which crystallize with discrete cations, anions, and solvent molecules. In (I), the complete decachlorocyclopentasilane ring is generated by a crystallographic twofold rotation axis. In (II), one cation is located on a general position and the other two are disordered about centres of inversion. These are the first structures featuring the structural motif of a five-membered cyclopentasilane ring coordinated from both sides by a chloride ion. The extended structures of (I) and (II) feature numerous C$\mathrm{H}$. . Cl interactions. In (II), the $\mathrm{N}$ atoms are located on centres of inversion and as a result, the ethylene chains are disordered over equally occupied orientations.

\section{Chemical context}

The title compounds are the first known halide diadducts of the long-known perchlorinated cyclopentasilane $\mathrm{Si}_{5} \mathrm{Cl}_{10}$ (Hengge \& Kovar, 1977). Their structures can be seen as inverse-sandwich complexes, in which two chloride ions lie above and below the planar five-membered silicon ring.

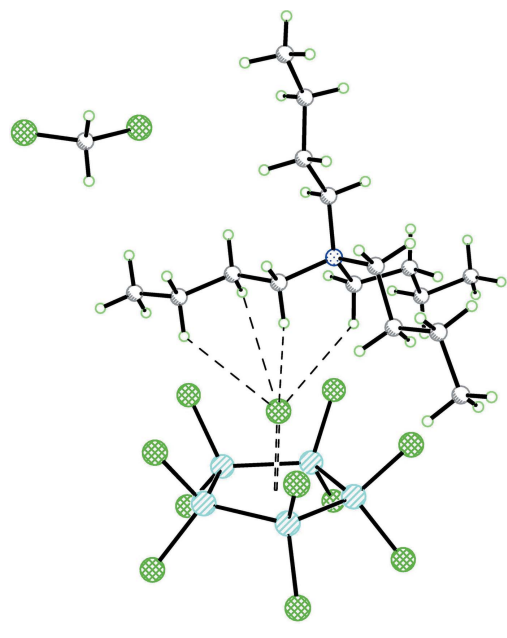

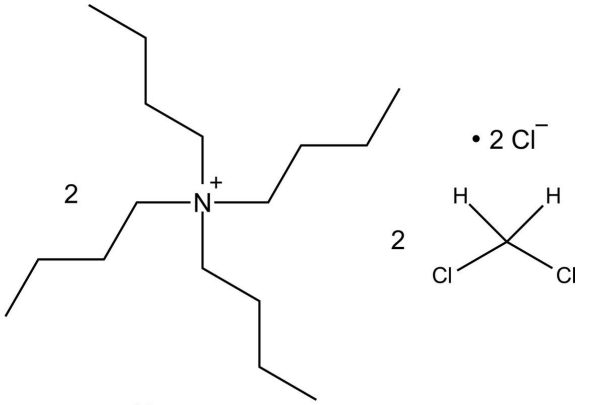

(I)<smiles>Cl[Si]1(Cl)[Si](Cl)(Cl)[Si](Cl)(Cl)[Si](Cl)(Cl)[Si]1(Cl)Cl</smiles>

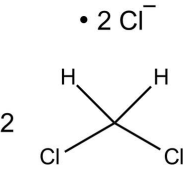

(II) 

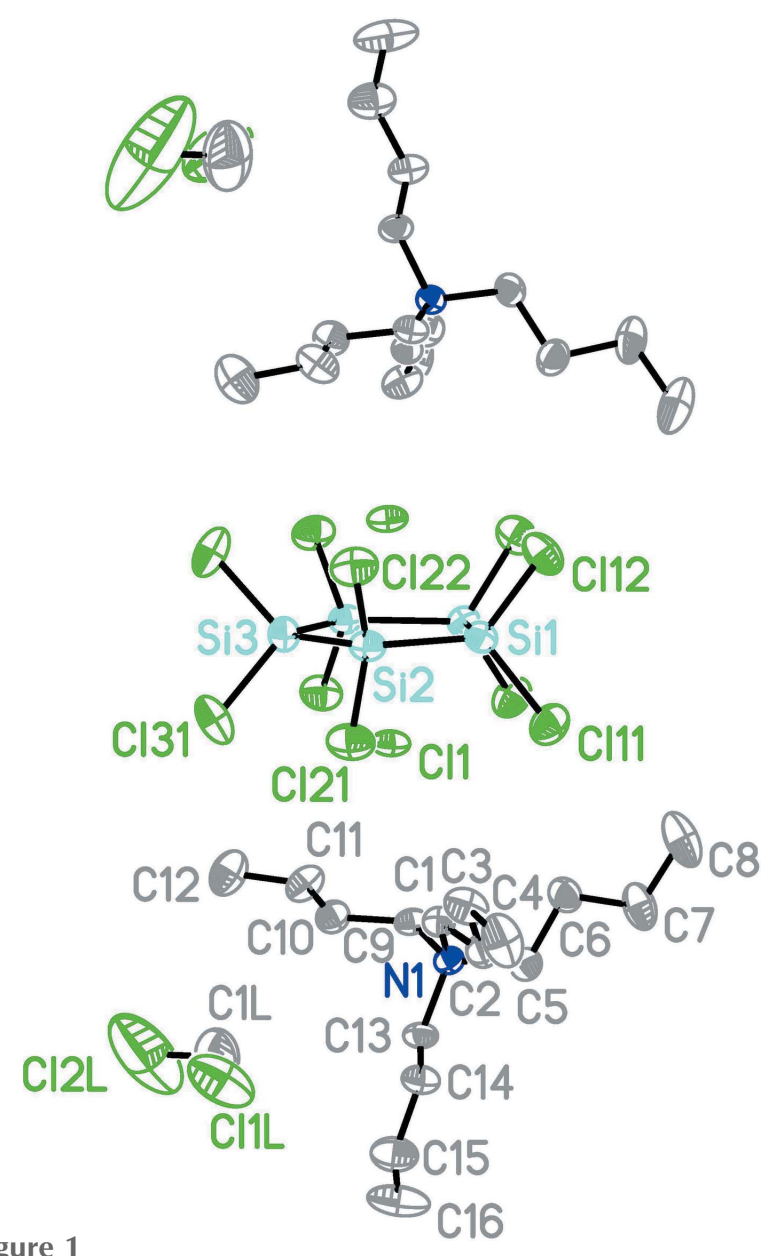

Figure 1

Perspective view of (I) with displacement ellipsoids drawn at the $50 \%$ probability level. For clarity, $\mathrm{H}$ atoms are omitted and only the symmetry independent molecules are labelled. Atoms without labels are generated by the symmetry operator $-x+1, y,-z+\frac{3}{2}$.

In the recent years, new and facile synthetic protocols for the $\mathrm{Cl}^{-}$diadduct $\left[\mathrm{Si}_{6} \mathrm{Cl}_{14}\right]^{2-}$ of dodecachlorocyclohexasilane have been developed. It can either be prepared through the chloride-induced disproportionation of $\mathrm{Si}_{2} \mathrm{Cl}_{6}$, which leads directly to $\left[\mathrm{Si}_{6} \mathrm{Cl}_{14}\right]^{2-}$ (Tillmann et al., 2012, 2014; Tillmann, Moxter et al., 2015), or the Lewis acidic uncomplexed $\mathrm{Si}_{6} \mathrm{Cl}_{12}$ can be used as the starting material. In the latter case, mere addition of soluble chloride salts $\left[R_{4} \mathrm{~N}\right] \mathrm{Cl}(R=n \mathrm{Bu}$ or Et $)$ leads to the formation of $\left[\mathrm{Si}_{6} \mathrm{Cl}_{14}\right]^{2-}$ (Dai et al., 2010). Given this background, it was of interest to investigate the Lewis acidity and ability of $\mathrm{Si}_{5} \mathrm{Cl}_{10}$ to bind $\mathrm{Cl}^{-}$ions.

\section{Structural commentary}

Bis(tetra- $n$-butylammonium) dichloride decachlorocyclopentasilane dichloromethane disolvate, $2 \mathrm{C}_{16} \mathrm{H}_{36} \mathrm{~N}^{+} \cdot 2 \mathrm{Cl}^{-}$.$\mathrm{Si}_{5} \mathrm{Cl}_{10} \cdot 2 \mathrm{CH}_{2} \mathrm{Cl}_{2}$, (I), crystallizes with discrete cations, anions, and solvent molecules (Fig. 1). The five-membered decachlorocyclopentasilane ring is located on a twofold rotation axis. The $\mathrm{Si}-\mathrm{Cl}$ bond lengths range from 2.081 (3) $\AA$ for $\mathrm{Si} 2-$ $\mathrm{Cl} 21$ to 2.100 (3) $\AA$ for $\mathrm{Si} 2-\mathrm{Cl} 22$. The $\mathrm{Si}-\mathrm{Si}$ bond lengths do not vary markedly: they range from 2.339 (3) $\AA$ ( $\mathrm{Si} 1-\mathrm{Si} 2)$ to 2.347 (3) $\AA$ ( $\mathrm{Si} 2-\mathrm{Si} 3)$.

The almost planar ring (r.m.s. deviation $0.002 \AA$ ) is coordinated by two chloride anions located above and below the ring. The distances of the chloride ions to the $\mathrm{Si}$ atoms

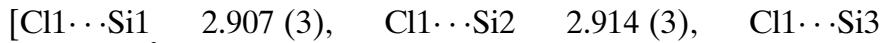
2.930 (3) $\AA$,] show that the chloride ions are located almost exactly above the centroid of the ring [distance $\mathrm{Cl} 1 \cdots \mathrm{Cg}=$ 2.1434 (16) А].

Bis(tetraethylammonium) dichloride decachlorocyclopentasilane dichloromethane disolvate, $2 \mathrm{C}_{8} \mathrm{H}_{20} \mathrm{~N}^{+} \cdot 2 \mathrm{Cl}^{-}$.. $\mathrm{Si}_{5} \mathrm{Cl}_{10} \cdot 2 \mathrm{CH}_{2} \mathrm{Cl}_{2}$, (II), crystallizes as (I) with discrete cations, anions, and solvent molecules (Fig. 2). The $\mathrm{Si}-\mathrm{Cl}$ bonds again lie in a quite narrow range [2.0805 (9) $\AA$ (Si1-Cl12) to $2.1102(8) \AA(\mathrm{Si} 2-\mathrm{Cl} 22)]$ and the $\mathrm{Si}-\mathrm{Si}$ bond lengths are also very similar $[2.3386(8) \AA(\mathrm{Si} 1-\mathrm{Si} 2)$ to 2.3473 (7) $\AA$ ( $\mathrm{Si} 4-$ Si5)].

The five-membered decachlorocyclopentasilane ring is almost planar (r.m.s. deviation $=0.017 \AA$ ) and coordinated by two chloride anions located above and below the ring with a $\mathrm{Cl} \cdots \mathrm{Cg}$ distance of 2.1781 (5) $\AA$ for $\mathrm{Cl} 1$ and 2.1237 (5) $\AA$ for $\mathrm{Cl} 2$. The $\mathrm{Cl}$ - . Si distances range from 2.9381 (7) $\AA$ (Cl1 ‥S Si5)

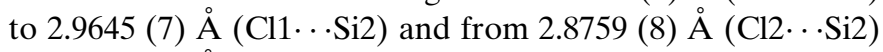

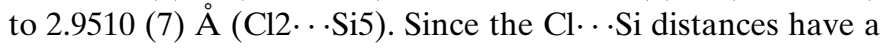
broader range for $\mathrm{Cl} 2$, it can be said that this ion is slightly displaced from a position directly over the ring centroid.

It is interesting to note that (I) and (II) have - apart from the different cations - the same molecular stoichiometry, i.e. one $\mathrm{Si}_{5} \mathrm{Cl}_{10}$ ring coordinated by two chloride anions, two cations and two solvent dichloromethane molecules. However, since (I) has twofold rotation symmetry, there are only half of the chemical entities in the asymmetric unit.

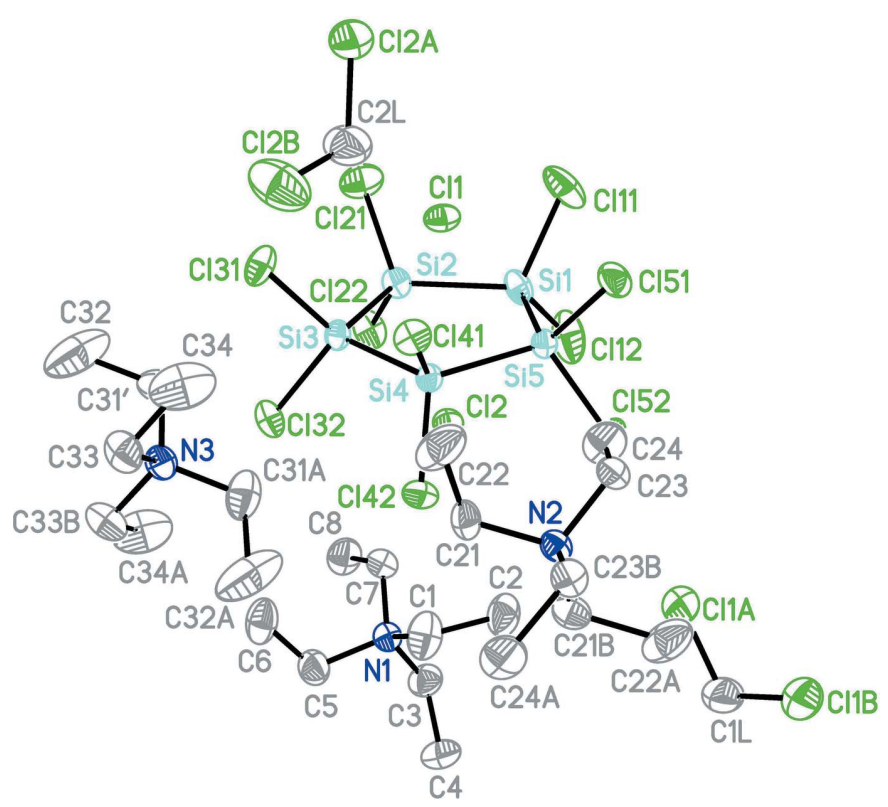

Figure 2

Perspective view of (II) with displacement ellipsoids drawn at the $50 \%$ probability level. For clarity, $\mathrm{H}$ atoms are omitted and only one of the two disordered sites of the tetraethylammonium cations are shown. 
Table 1

Hydrogen-bond geometry $\left(\AA{ }^{\circ}\right)$ for $(\mathrm{I})$.

\begin{tabular}{lllll}
\hline$D-\mathrm{H} \cdots A$ & $D-\mathrm{H}$ & $\mathrm{H} \cdots A$ & $D \cdots A$ & $D-\mathrm{H} \cdots A$ \\
\hline $\mathrm{C} 1-\mathrm{H} 1 B \cdots \mathrm{Cl} 1$ & 0.99 & 2.88 & $3.686(7)$ & 139 \\
$\mathrm{C} 2-\mathrm{H} 2 A \cdots \mathrm{Cl} 31^{\mathrm{i}}$ & 0.99 & 2.89 & $3.596(8)$ & 129 \\
$\mathrm{C} 5-\mathrm{H} 5 A \cdots \mathrm{Cl} 22^{\mathrm{ii}}$ & 0.99 & 2.99 & $3.945(7)$ & 163 \\
$\mathrm{C} 9-\mathrm{H} 9 B \cdots \mathrm{Cl} 1$ & 0.99 & 2.91 & $3.652(7)$ & 132 \\
$\mathrm{C} 1 L-\mathrm{H} 1 L 1 \cdots \mathrm{Cl} 12^{\mathrm{ii}}$ & 0.99 & 2.96 & $3.528(13)$ & 119 \\
\hline
\end{tabular}

Symmetry codes: (i) $-x+1,-y+1,-z+1$; (ii) $x-\frac{1}{2},-y+\frac{3}{2}, z-\frac{1}{2}$.

Table 2

Hydrogen-bond geometry $\left(\AA{ }^{\circ}{ }^{\circ}\right)$ for (II).

\begin{tabular}{lllll}
\hline$D-\mathrm{H} \cdots A$ & $D-\mathrm{H}$ & $\mathrm{H} \cdots A$ & $D \cdots A$ & $D-\mathrm{H} \cdots A$ \\
\hline $\mathrm{C} 1-\mathrm{H} 1 B \cdots \mathrm{Cl} 42$ & 0.99 & 2.99 & $3.829(3)$ & 144 \\
$\mathrm{C} 2-\mathrm{H} 2 C \cdots \mathrm{Cl} 2$ & 0.98 & 2.95 & $3.753(3)$ & 139 \\
$\mathrm{C} 3-\mathrm{H} 3 A \cdots \mathrm{Cl} 52^{\mathrm{i}}$ & 0.99 & 2.79 & $3.643(3)$ & 144 \\
$\mathrm{C} 3-\mathrm{H} 3 B \cdots \mathrm{Cl} 2 B^{\text {ii }}$ & 0.99 & 2.98 & $3.804(3)$ & 142 \\
$\mathrm{C} 5-\mathrm{H} 5 B \cdots \mathrm{Cl} 22^{\text {iii }}$ & 0.99 & 2.89 & $3.850(3)$ & 165 \\
$\mathrm{C} 6-\mathrm{H} 6 B \cdots \mathrm{Cl} 21^{\text {iii }}$ & 0.98 & 2.86 & $3.630(3)$ & 136 \\
$\mathrm{C} 7-\mathrm{H} 7 A \cdots \mathrm{Cl} 2$ & 0.99 & 2.86 & $3.394(2)$ & 115 \\
$\mathrm{C} 22-\mathrm{H} 22 C \cdots \mathrm{Cl} 51^{\text {iv }}$ & 0.98 & 2.89 & $3.847(4)$ & 165 \\
$\mathrm{C} 22-\mathrm{H} 22 E \cdots \mathrm{Cl} 41$ & 0.98 & 2.90 & $3.859(3)$ & 165 \\
$\mathrm{C} 23-\mathrm{H} 23 B \cdots \mathrm{Cl} 1^{\text {iv }}$ & 0.99 & 2.98 & $3.465(4)$ & 111 \\
$\mathrm{C} 23^{\prime}-\mathrm{H} 23 C \cdots \mathrm{Cl} 42$ & 0.99 & 2.87 & $3.497(4)$ & 122 \\
$\mathrm{C} 24-\mathrm{H} 24 C \cdots \mathrm{Cl} 41$ & 0.98 & 2.84 & $3.793(3)$ & 164 \\
$\mathrm{C} 24-\mathrm{H} 24 E \cdots \mathrm{Cl} 51^{\text {iv }}$ & 0.98 & 2.81 & $3.771(3)$ & 165 \\
$\mathrm{C} 24-\mathrm{H} 24 F \cdots \mathrm{Cl} 2 A^{\text {iv }}$ & 0.98 & 2.92 & $3.778(3)$ & 147 \\
$\mathrm{C} 31^{\prime}-\mathrm{H} 31 C \cdots \mathrm{Cl} 31$ & 0.99 & 2.95 & $3.434(5)$ & 111 \\
$\mathrm{C} 32-\mathrm{H} 32 F \cdots \mathrm{Cl} 21^{\mathrm{v}}$ & 0.98 & 2.76 & $3.584(4)$ & 142 \\
$\mathrm{C} 33-\mathrm{H} 33 A \cdots \mathrm{Cl} 32^{\text {vi }}$ & 0.99 & 2.94 & $3.515(4)$ & 118 \\
$\mathrm{C} 33^{\prime}-\mathrm{H} 33 D \cdots \mathrm{Cl} 41$ & 0.99 & 2.98 & $3.630(5)$ & 124 \\
$\mathrm{C} 34-\mathrm{H} 34 A \cdots \mathrm{Cl} 1 B^{\text {vii }}$ & 0.98 & 2.93 & $3.556(3)$ & 123 \\
$\mathrm{C} 34-\mathrm{H} 34 C \cdots \mathrm{Cl} 2 B$ & 0.98 & 2.89 & $3.716(4)$ & 142 \\
$\mathrm{C} 34-\mathrm{H} 34 A \cdots \mathrm{Cl} 1 B^{\text {vii }}$ & 0.98 & 2.93 & $3.556(3)$ & 123 \\
$\mathrm{C} 1 L-\mathrm{H} 1 L 1 \cdots \mathrm{Cl} 12^{\mathrm{i}}$ & 0.99 & 2.90 & $3.421(3)$ & 114 \\
$\mathrm{C} 2 L-\mathrm{H} 2 L 2 \cdots \mathrm{Cl} 41$ & 0.99 & 2.96 & $3.465(3)$ & 113 \\
\hline
\end{tabular}

Symmetry codes: (i) $-x+1,-y+1,-z$; (ii) $x, y, z-1$; (iii) $x-1, y, z$; (iv) $-x+1,-y+1,-z+1 ; \quad$ (v) $-x+1,-y+2,-z+1$; (vi) $-x,-y+2,-z+1$; (vii) $-x,-y+1,-z+1$.

\section{Supramolecular features}

The components of (I) and (II) are linked by a plethora of $\mathrm{C}-$ $\mathrm{H}$... Cl contacts (Tables 1 and 2, respectively); in particular the chloride ions are surrounded by $\mathrm{C}-\mathrm{H}$ groups. For an example, see Fig. 3. As a result of the disorder of the N2 and N3 cations in (II), a plot showing the coordination of the $\mathrm{Cl}$ ions looks extremely crowded and is therefore omitted.

\section{Database survey}

The present structures are the first examples of a decachlorocyclopentasilane ring coordinated by two anions. There are only two structures of a decachlorocyclopentasilane ring in the CSD (Version 5.38 of November 2016 plus three updates; Groom et al., 2016), namely decachlorocyclopentasilane 4methylbenzonitrile solvate (refcode ELAFON; Dai et al., 2010) and decachlorocyclopentasilane acetonitrile solvate (ELAFIH; Dai et al., 2010). In both of them, the decachlorocyclopentasilane ring is almost planar $(0.017 \AA$ for ELAFON and $0.001 \AA$ for ELAFIH) and shows almost no
Table 3

Mean values $(\AA)$ of $\mathrm{Si}-\mathrm{Si}, \mathrm{Si}-\mathrm{Cl}$ bond lengths and $\mathrm{Cl} / \mathrm{N} \cdots C g$ contacts in the title compounds and related structures.

$X=\mathrm{Cl}$ for (I) and (II) and $X=\mathrm{N}$ for ELAFON and ELAFIH. The row for $\mathrm{Si}_{6} \mathrm{Cl}_{12}$ contains data for dichloride dodecachlorohexasilanes (Tillmann, Lerner \& Bolte, 2015).

\begin{tabular}{llll}
\hline Structure & $\mathrm{Si}-\mathrm{Si}$ & $\mathrm{Si}-\mathrm{Cl}$ & $X \cdots C g$ \\
\hline (I) & 2.342 & 2.092 & 2.143 \\
(II) & 2.344 & 2.092 & 2.151 \\
ELAFON & 2.363 & 2.049 & 2.174 \\
ELAFIH & 2.348 & 2.036 & 2.234 \\
$\mathrm{Si}_{6} \mathrm{Cl}_{12}$ & 2.322 & 2.078 & 1.90 \\
\hline
\end{tabular}

variation in the $\mathrm{Si}-\mathrm{Si}(2.358-2.368 \AA$ for ELAFON and 2.342-2.349 $\AA$ for ELAFIH) and $\mathrm{Si}-\mathrm{Cl}(2.030-2.059 \AA$ for ELAFON and 2.034- 2.038 $\AA$ for ELAFIH) bond lengths.

The distance of the $\mathrm{N}$ atom to the centroid of the ring is 2.152 and $2.196 \AA$ for ELAFON and $2.234 \AA$ for ELAFIH. This difference could be due to the steric demand of the benzene ring in ELAFIH. The $\mathrm{N} \cdots C g$ distances are in the same range as the $\mathrm{Cl} \cdots \mathrm{Cg}$ distances in (I) and (II).

Mean values of the structural parameters of the four compared structures and dichloride dodecachlorocyclohexasilanes (Tillmann, Lerner \& Bolte, 2015) are compiled in Table 3. It is remarkable that the $\mathrm{Si}-\mathrm{Si}$ and $\mathrm{Si}-\mathrm{Cl}$ bond

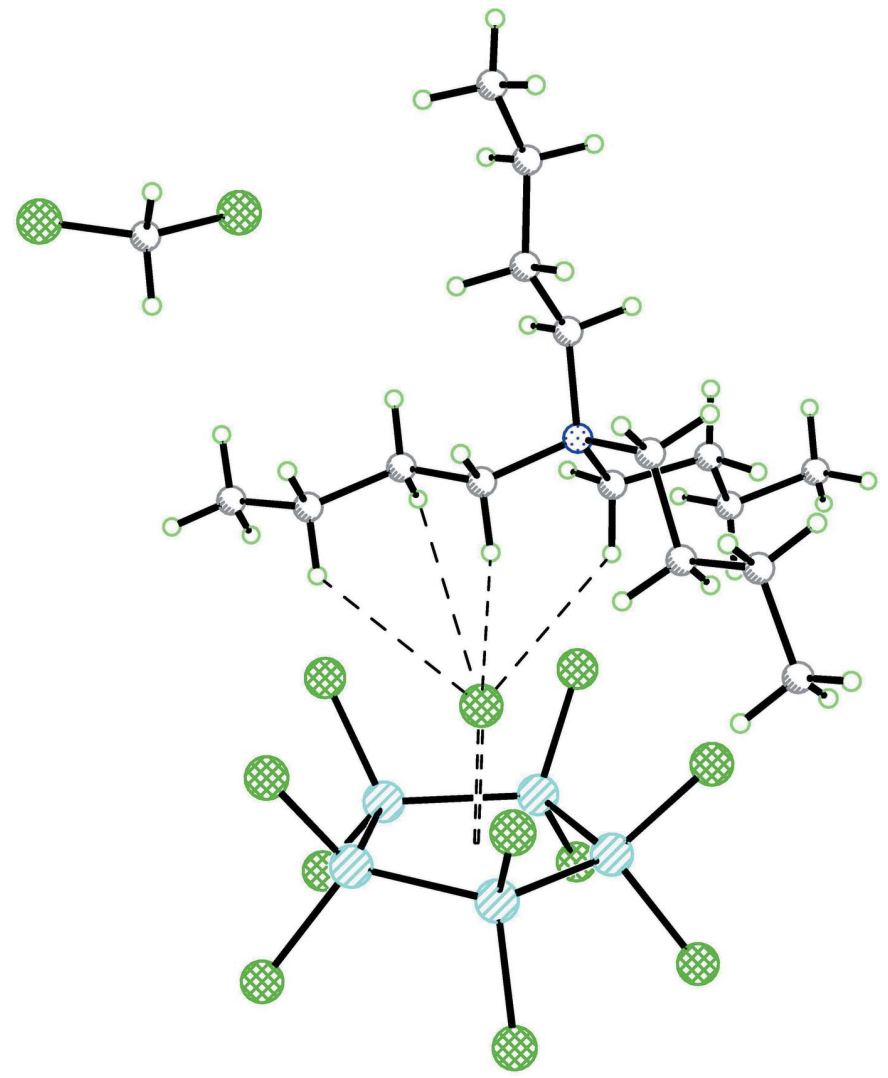

Figure 3

Perspective view of (I) showing the environment of the $\mathrm{Cl}$ anion. The contact to the centre of the five-membered ring is drawn as an open dashed bond. $\mathrm{H} \cdots \mathrm{Cl}$ contacts less than $3.5 \AA$ are drawn as dashed lines. 
Table 4

Experimental details.

(I)

Crystal data

Chemical formula

$M_{\mathrm{r}}$

Crystal system, space group

Temperature (K)

$a, b, c(\AA)$

$\alpha, \beta, \gamma\left({ }^{\circ}\right)$

$V\left(\AA^{3}\right)$

$Z$

Radiation type

$\mu\left(\mathrm{mm}^{-1}\right)$

Crystal size (mm)

Data collection

Diffractometer

Absorption correction

$T_{\min }, T_{\max }$

No. of measured, independent and

observed $[I>2 \sigma(I)]$ reflections

$R_{\text {int }}$

$(\sin \theta / \lambda)_{\max }\left(\AA^{-1}\right)$

Refinement

$R\left[F^{2}>2 \sigma\left(F^{2}\right)\right], w R\left(F^{2}\right), S$

No. of reflections

No. of parameters

$\mathrm{H}$-atom treatment

$\Delta \rho_{\max }, \Delta \rho_{\min }\left(\mathrm{e} \AA^{-3}\right)$

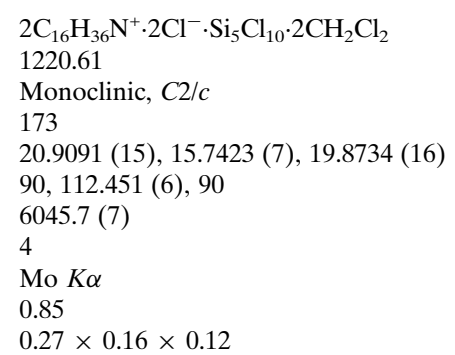

(II)

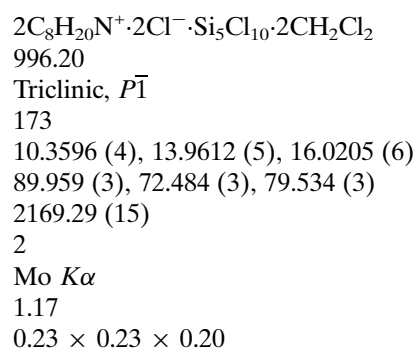

Stoe IPDS II two-circle

Multi-scan ( $X$-AREA; Stoe \& Cie, 2001)

$0.543,1.000$

$32650,5699,4428$

Stoe IPDS II two-circle

Multi-scan ( $X$-AREA; Stoe \& Cie, 2001)

$0.408,1.000$

62962, 13044, 11976

0.060

0.043

0.715

$0.103,0.199,1.15$

5699

258

$\mathrm{H}$-atom parameters constrained $1.50,-1.46$
$0.048,0.131,1.08$

13044

409

$\mathrm{H}$-atom parameters constrained $0.87,-0.84$

Computer programs: X-AREA (Stoe \& Cie, 2001), XP in SHELXTL-Plus and SHELXS97 (Sheldrick, 2008), SHELXL2014 (Sheldrick, 2015) and publCIF (Westrip, 2010).

lengths do not vary significantly between the five and sixmembered Si rings, but the $\mathrm{Cl} \cdots \mathrm{Cg}$ distance in the dodecachlorocyclohexasilanes is significantly shorter than for decachlorocyclopentasilane. This might be due to the fact that the $\mathrm{Cl}$ ligands form a narrower cone in five- compared to sixmembered rings.

\section{Synthesis and crystallization}

The addition of a solution of $\left[R_{4} \mathrm{~N}\right] \mathrm{Cl}(R=n \mathrm{Bu}$ or $\mathrm{Et})$ in $\mathrm{CH}_{2} \mathrm{Cl}_{2}$ at $195 \mathrm{~K}$ to a solution of $\mathrm{Si}_{5} \mathrm{Cl}_{10}$ in $\mathrm{CH}_{2} \mathrm{Cl}_{2}$ furnished the $\mathrm{Cl}^{-}$diadducts $\left[R_{4} \mathrm{~N}\right]_{2}\left[\mathrm{Si}_{5} \mathrm{Cl}_{12}\right](R=n \mathrm{Bu}$ or Et) (Fig. 4). Crystals of $\left[R_{4} \mathrm{~N}\right]_{2}\left[\mathrm{Si}_{5} \mathrm{Cl}_{12}\right](R=n \mathrm{Bu}$ or Et $)$ could be harvested after storage of the reaction solution for one week at $195 \mathrm{~K}$ in $89 \%$ and $93 \%$ yield, respectively. Both adducts are stable in the solid phase under inert conditions. However, in solution a rapid transformation of $\left[n \mathrm{Bu}_{4} \mathrm{~N}\right]_{2}\left[\mathrm{Si}_{5} \mathrm{Cl}_{12}\right]$ to $\left[n \mathrm{Bu}_{4} \mathrm{~N}\right]_{2}\left[\mathrm{Si}_{6} \mathrm{Cl}_{14}\right]$ and $\left[n \mathrm{Bu}_{4} \mathrm{~N}_{2}\left[\mathrm{Si}_{7} \mathrm{Cl}_{16}\right]\right.$ (Fig. 5) can be observed via ${ }^{29} \mathrm{Si} \mathrm{NMR}$ spectroscopy (for the NMR spectrum see Fig. S1 in the Supporting information), while $\left[\mathrm{Et}_{4} \mathrm{~N}\right]_{2}\left[\mathrm{Si}_{5} \mathrm{Cl}_{12}\right]$ is not soluble.
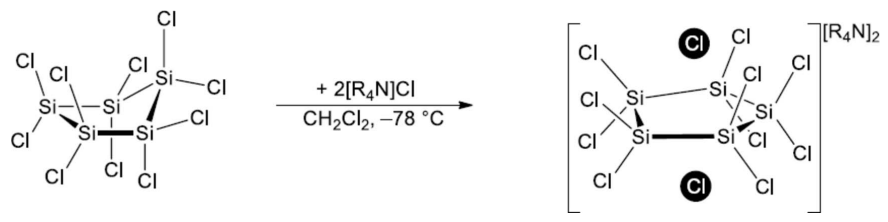

Figure 4

Synthesis of $\left[R_{4} \mathrm{~N}\right]_{2}\left[\mathrm{Si}_{5} \mathrm{Cl}_{12}\right](R=n \mathrm{Bu}$ or $\mathrm{Et})$.
For comparison, a ${ }^{29} \mathrm{Si}$ CP/MAS NMR spectrum of single crystals of $\left[n \mathrm{Bu}_{4} \mathrm{~N}\right]_{2}\left[\mathrm{Si}_{5} \mathrm{Cl}_{12}\right]$ was recorded (Fig. S2 in the Supporting information).

\section{Refinement details}

Crystal data, data collection and structure refinement details are summarized in Table $4 . \mathrm{H}$ atoms were refined using a riding model, with $\mathrm{C}_{\text {methyl }}-\mathrm{H}=0.98 \AA$ or $\mathrm{C}_{\text {methylene }}-\mathrm{H}=$ $0.99 \AA$ and with $U_{\text {iso }}(\mathrm{H})=1.5 U_{\text {eq }}\left(\mathrm{C}_{\text {methyl }}\right)$ or $1.2 U_{\text {eq }}(\mathrm{C})$.

The $\mathrm{Cl}$ atoms of the dichloromethane solvent molecule in (I) have rather large displacement ellipsoids, but since no valid disorder model for splitting this molecule could be found, refinement with enlarged ADPs was preferred. In (II), atoms $\mathrm{N} 2$ and N3 are located on centres of inversion. As a result, the ethylene chains are disordered over equally occupied orientations.

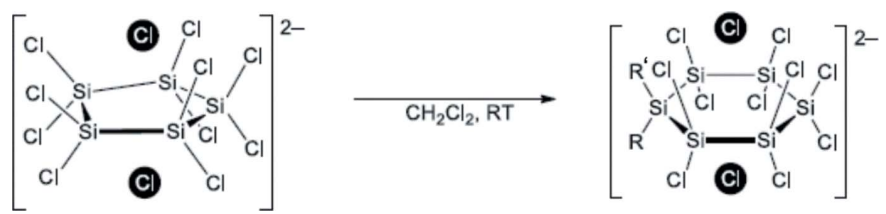

Figure 5

Transformation of $\left[n \mathrm{Bu}_{4} \mathrm{~N}\right]_{2}\left[\mathrm{Si}_{5} \mathrm{Cl}_{12}\right]$ (I) to $\left[n \mathrm{Bu}_{4} \mathrm{~N}\right]_{2}\left[\mathrm{Si}_{6} \mathrm{Cl}_{14}\right]\left(R=R^{\prime}=\mathrm{Cl}\right)$ and $\left[n \mathrm{Bu}_{4} \mathrm{~N}\right]_{2}\left[\mathrm{Si}_{7} \mathrm{Cl}_{16}\right]\left(R=\mathrm{Cl} ; R^{\prime}=\mathrm{SiCl}_{3}\right)$. 


\section{Acknowledgements}

The authors wish to thank Johanna Becker-Baldus for the recording of the ${ }^{29} \mathrm{Si} \mathrm{CP} / \mathrm{MAS}$ NMR spectrum.

\section{References}

Dai, X., Anderson, K. J., Schulz, D. L. \& Boudjouk, P. (2010). Dalton Trans. 39, 11188-11192.

Groom, C. R., Bruno, I. J., Lightfoot, M. P. \& Ward, S. C. (2016). Acta Cryst. B72, 171-179.

Hengge, E. \& Kovar, D. (1977). J. Organomet. Chem. 125, C29-C32.

Sheldrick, G. M. (2008). Acta Cryst. A64, 112-122.
Sheldrick, G. M. (2015). Acta Cryst. C71, 3-8.

Stoe \& Cie (2001). $X$-AREA and $X$-RED32. Stoe \& Cie, Darmstadt, Germany.

Tillmann, J., Lerner, H.-W. \& Bolte, M. (2015). Acta Cryst. C71, 883888.

Tillmann, J., Meyer, L., Schweizer, J. I., Bolte, M., Lerner, H.-W., Wagner, M. \& Holthausen, M. C. (2014). Chem. Eur. J. 20, 92349239.

Tillmann, J., Meyer-Wegner, F., Nadj, A., Becker-Baldus, J., Sinke, T., Bolte, M., Holthausen, M. C., Wagner, M. \& Lerner, H.-W. (2012). Inorg. Chem. 51, 8599-8606.

Tillmann, J., Moxter, M., Bolte, M., Lerner, H.-W. \& Wagner, M. (2015). Inorg. Chem. 54, 9611-9618.

Westrip, S. P. (2010). J. Appl. Cryst. 43, 920-925. 


\section{supporting information}

Acta Cryst. (2017). E73, 1903-1907 [https://doi.org/10.1107/S2056989017016310]

Decachlorocyclopentasilanes coordinated by pairs of chloride anions, with different cations, but the same solvent molecules

Maximilian Moxter, Julian Teichmann, Hans-Wolfram Lerner, Michael Bolte and Matthias

Wagner

Computing details

For both structures, data collection: $X$-AREA (Stoe \& Cie, 2001); cell refinement: $X$-AREA (Stoe \& Cie, 2001); data reduction: X-AREA (Stoe \& Cie, 2001); program(s) used to solve structure: SHELXS97 (Sheldrick, 2008); program(s) used to refine structure: SHELXL2014 (Sheldrick, 2015); molecular graphics: XP in SHELXTL-Plus (Sheldrick, 2008); software used to prepare material for publication: SHELXL2014 (Sheldrick, 2015) and publCIF (Westrip, 2010).

Bis(tetra-n-butylammonium) dichloride decachlorocyclopentasilane dichloromethane disolvate (I)

Crystal data

$2 \mathrm{C}_{16} \mathrm{H}_{36} \mathrm{~N}^{+} \cdot 2 \mathrm{Cl}^{-} \cdot \mathrm{Si}_{5} \mathrm{Cl}_{10} \cdot 2 \mathrm{CH}_{2} \mathrm{Cl}_{2}$

$M_{r}=1220.61$

Monoclinic, $C 2 / c$

$a=20.9091(15) \AA$

$b=15.7423(7) \AA$

$c=19.8734(16) \AA$

$\beta=112.451(6)^{\circ}$

$V=6045.7(7) \AA^{3}$

$Z=4$

Data collection

Stoe IPDS II two-circle diffractometer

Radiation source: Genix 3D I $\mu$ S microfocus Xray source

$\omega$ scans

Absorption correction: multi-scan

(X-AREA; Stoe \& Cie, 2001)

$T_{\min }=0.543, T_{\max }=1.000$

Refinement

Refinement on $F^{2}$

Least-squares matrix: full

$R\left[F^{2}>2 \sigma\left(F^{2}\right)\right]=0.103$

$w R\left(F^{2}\right)=0.199$

$S=1.15$

5699 reflections

258 parameters

0 restraints
$F(000)=2544$

$D_{\mathrm{x}}=1.341 \mathrm{Mg} \mathrm{m}^{-3}$

Mo $K \alpha$ radiation, $\lambda=0.71073 \AA$

Cell parameters from 30920 reflections

$\theta=3.3-25.8^{\circ}$

$\mu=0.85 \mathrm{~mm}^{-1}$

$T=173 \mathrm{~K}$

Needle, colourless

$0.27 \times 0.16 \times 0.12 \mathrm{~mm}$

32650 measured reflections

5699 independent reflections

4428 reflections with $I>2 \sigma(I)$

$R_{\text {int }}=0.060$

$\theta_{\max }=25.8^{\circ}, \theta_{\min }=3.3^{\circ}$

$h=-25 \rightarrow 25$

$k=-18 \rightarrow 19$

$l=-24 \rightarrow 24$

Hydrogen site location: inferred from neighbouring sites

$\mathrm{H}$-atom parameters constrained

$w=1 /\left[\sigma^{2}\left(F_{\mathrm{o}}{ }^{2}\right)+(0.0209 P)^{2}+98.7944 P\right]$

where $P=\left(F_{\mathrm{o}}{ }^{2}+2 F_{\mathrm{c}}{ }^{2}\right) / 3$

$(\Delta / \sigma)_{\max }<0.001$

$\Delta \rho_{\max }=1.50 \mathrm{e} \AA^{-3}$

$\Delta \rho_{\min }=-1.46$ e $\AA^{-3}$ 


\section{Special details}

Geometry. All esds (except the esd in the dihedral angle between two 1.s. planes) are estimated using the full covariance matrix. The cell esds are taken into account individually in the estimation of esds in distances, angles and torsion angles; correlations between esds in cell parameters are only used when they are defined by crystal symmetry. An approximate (isotropic) treatment of cell esds is used for estimating esds involving l.s. planes.

Fractional atomic coordinates and isotropic or equivalent isotropic displacement parameters $\left(\hat{A}^{2}\right)$

\begin{tabular}{|c|c|c|c|c|}
\hline & $x$ & $y$ & $z$ & $U_{\text {iso }} * / U_{\text {eq }}$ \\
\hline $\mathrm{Cl1}$ & $0.44920(9)$ & $0.65838(13)$ & $0.63371(9)$ & $0.0392(4)$ \\
\hline Sil & $0.55353(9)$ & $0.75962(13)$ & $0.74394(10)$ & $0.0312(4)$ \\
\hline $\mathrm{Si} 2$ & $0.58692(10)$ & $0.61853(14)$ & $0.73977(10)$ & $0.0329(4)$ \\
\hline $\mathrm{Si} 3$ & 0.5000 & $0.53144(19)$ & 0.7500 & $0.0368(7)$ \\
\hline Cl11 & $0.55030(11)$ & $0.83349(15)$ & $0.65484(12)$ & $0.0536(5)$ \\
\hline $\mathrm{Cl12}$ & $0.62942(10)$ & $0.82642(15)$ & $0.82814(12)$ & $0.0535(6)$ \\
\hline $\mathrm{Cl} 21$ & $0.60720(11)$ & $0.58866(16)$ & $0.64782(11)$ & $0.0534(6)$ \\
\hline $\mathrm{Cl} 22$ & $0.68493(9)$ & $0.59548(15)$ & $0.82099(11)$ & $0.0499(5)$ \\
\hline $\mathrm{Cl} 31$ & $0.46207(13)$ & $0.44593(15)$ & $0.66352(15)$ & $0.0667(7)$ \\
\hline N1 & $0.3868(3)$ & $0.7205(4)$ & $0.4082(3)$ & $0.0278(12)$ \\
\hline $\mathrm{C} 1$ & $0.4614(3)$ & $0.6993(5)$ & $0.4564(4)$ & $0.0309(15)$ \\
\hline $\mathrm{H} 1 \mathrm{~A}$ & 0.4668 & 0.6368 & 0.4578 & $0.037^{*}$ \\
\hline H1B & 0.4699 & 0.7185 & 0.5065 & $0.037^{*}$ \\
\hline $\mathrm{C} 2$ & $0.5172(4)$ & $0.7380(5)$ & $0.4335(4)$ & $0.0373(17)$ \\
\hline $\mathrm{H} 2 \mathrm{~A}$ & 0.5102 & 0.7188 & 0.3838 & $0.045^{*}$ \\
\hline $\mathrm{H} 2 \mathrm{~B}$ & 0.5135 & 0.8008 & 0.4329 & $0.045^{*}$ \\
\hline $\mathrm{C} 3$ & $0.5881(4)$ & $0.7115(6)$ & $0.4859(4)$ & $0.047(2)$ \\
\hline $\mathrm{H} 3 \mathrm{~A}$ & 0.5894 & 0.6489 & 0.4913 & $0.056^{*}$ \\
\hline H3B & 0.5969 & 0.7367 & 0.5343 & $0.056^{*}$ \\
\hline $\mathrm{C} 4$ & $0.6455(4)$ & $0.7395(6)$ & $0.4601(6)$ & $0.060(3)$ \\
\hline $\mathrm{H} 4 \mathrm{~A}$ & 0.6905 & 0.7210 & 0.4956 & $0.090^{*}$ \\
\hline H4B & 0.6375 & 0.7137 & 0.4127 & $0.090^{*}$ \\
\hline $\mathrm{H} 4 \mathrm{C}$ & 0.6450 & 0.8015 & 0.4557 & $0.090^{*}$ \\
\hline $\mathrm{C} 5$ & $0.3758(4)$ & $0.8156(5)$ & $0.3999(4)$ & $0.0351(16)$ \\
\hline $\mathrm{H} 5 \mathrm{~A}$ & 0.3258 & 0.8265 & 0.3728 & $0.042 *$ \\
\hline H5B & 0.4007 & 0.8374 & 0.3700 & $0.042 *$ \\
\hline C6 & $0.3994(4)$ & $0.8659(5)$ & $0.4706(4)$ & 0.0440 (19) \\
\hline H6A & 0.3744 & 0.8457 & 0.5010 & $0.053^{*}$ \\
\hline H6B & 0.4495 & 0.8568 & 0.4981 & $0.053^{*}$ \\
\hline C7 & $0.3852(4)$ & $0.9597(5)$ & $0.4545(5)$ & $0.055(2)$ \\
\hline H7A & 0.3348 & 0.9685 & 0.4288 & $0.066^{*}$ \\
\hline H7B & 0.4082 & 0.9787 & 0.4219 & $0.066^{*}$ \\
\hline $\mathrm{C} 8$ & $0.4110(5)$ & $1.0135(6)$ & $0.5234(6)$ & 0.078 \\
\hline H8A & 0.4008 & 1.0734 & 0.5104 & $0.116^{*}$ \\
\hline H8B & 0.3877 & 0.9957 & 0.5554 & $0.116^{*}$ \\
\hline $\mathrm{H} 8 \mathrm{C}$ & 0.4611 & 1.0059 & 0.5485 & $0.116^{*}$ \\
\hline C9 & 0.3385 (3) & $0.6853(5)$ & 0.4418 & $0.0303(15)$ \\
\hline H9A & 0.2910 & 0.7045 & 0.4120 & $0.036^{*}$ \\
\hline H9B & 0.3513 & 0.7107 & 0.4908 & $0.036^{*}$ \\
\hline
\end{tabular}




$\begin{array}{lllll}\text { C10 } & 0.3372(4) & 0.5894(5) & 0.4494(4) & 0.0396(17) \\ \text { H10A } & 0.3273 & 0.5623 & 0.4015 & 0.047^{*} \\ \text { H10B } & 0.3830 & 0.5693 & 0.4835 & 0.047^{*} \\ \text { C11 } & 0.2824(4) & 0.5642(5) & 0.4778(4) & 0.044(2) \\ \text { H11A } & 0.2364 & 0.5815 & 0.4420 & 0.053^{*} \\ \text { H11B } & 0.2906 & 0.5951 & 0.5237 & 0.053^{*} \\ \text { C12 } & 0.2818(4) & 0.4698(6) & 0.4915(5) & 0.059(2) \\ \text { H12A } & 0.2455 & 0.4567 & 0.5097 & 0.088^{*} \\ \text { H12B } & 0.2727 & 0.4389 & 0.4459 & 0.088^{*} \\ \text { H12C } & 0.3268 & 0.4525 & 0.5276 & 0.088^{*} \\ \text { C13 } & 0.3714(4) & 0.6817(5) & 0.3333(3) & 0.0348(16) \\ \text { H13A } & 0.4026 & 0.7085 & 0.3125 & 0.042^{*} \\ \text { H13B } & 0.3834 & 0.6206 & 0.3400 & 0.042^{*} \\ \text { C14 } & 0.2978(4) & 0.6896(6) & 0.2779(4) & 0.0412(18) \\ \text { H14A } & 0.2839 & 0.7501 & 0.2711 & 0.049^{*} \\ \text { H14B } & 0.2657 & 0.6589 & 0.2953 & 0.049^{*} \\ \text { C15 } & 0.2940(4) & 0.6515(7) & 0.2051(4) & 0.057(2) \\ \text { H15A } & 0.3294 & 0.6789 & 0.1907 & 0.068^{*} \\ \text { H15B } & 0.3049 & 0.5901 & 0.2118 & 0.068^{*} \\ \text { C16 } & 0.2249(5) & 0.6627(9) & 0.1459(5) & 0.083(4) \\ \text { H16A } & 0.2251 & 0.6375 & 0.1009 & 0.124^{*} \\ \text { H16B } & 0.1898 & 0.6346 & 0.1594 & 0.124^{*} \\ \text { H16C } & 0.2143 & 0.7234 & 0.1383 & 0.124^{*} \\ \text { C1L } & 0.1235(8) & 0.4569(8) & 0.2807(9) & 0.110(5) \\ \text { H1L1 } & 0.1317 & 0.4857 & 0.3275 & 0.132^{*} \\ \text { H1L2 } & 0.0904 & 0.4924 & 0.2416 & 0.132^{*} \\ \text { C11L } & 0.19998(18) & 0.4539(3) & 0.2681(2) & 0.1200(15) \\ \text { C12L } & 0.0881(5) & 0.3685(5) & 0.2810(5) & 0.294(6) \\ & & & & \end{array}$

Atomic displacement parameters $\left(\AA^{2}\right)$

\begin{tabular}{lllllll}
\hline & $U^{11}$ & $U^{22}$ & $U^{33}$ & $U^{12}$ & $U^{13}$ & $U^{23}$ \\
\hline C11 & $0.0321(9)$ & $0.0584(12)$ & $0.0230(8)$ & $-0.0037(9)$ & $0.0059(7)$ & $-0.0024(8)$ \\
Si1 & $0.0233(9)$ & $0.0364(11)$ & $0.0327(10)$ & $-0.0031(8)$ & $0.0092(8)$ & $-0.0029(8)$ \\
Si2 & $0.0281(9)$ & $0.0417(12)$ & $0.0302(10)$ & $0.0050(9)$ & $0.0124(8)$ & $-0.0018(9)$ \\
Si3 & $0.0373(15)$ & $0.0330(16)$ & $0.0421(16)$ & 0.000 & $0.0174(13)$ & 0.000 \\
C111 & $0.0498(11)$ & $0.0575(14)$ & $0.0576(13)$ & $-0.0087(10)$ & $0.0251(10)$ & $0.0109(11)$ \\
C112 & $0.0345(10)$ & $0.0572(14)$ & $0.0600(13)$ & $-0.0137(9)$ & $0.0082(9)$ & $-0.0193(11)$ \\
C121 & $0.0526(12)$ & $0.0739(15)$ & $0.0441(11)$ & $0.0101(11)$ & $0.0302(10)$ & $-0.0056(10)$ \\
C122 & $0.0276(9)$ & $0.0708(15)$ & $0.0470(11)$ & $0.0139(9)$ & $0.0095(8)$ & $0.0056(10)$ \\
C131 & $0.0663(15)$ & $0.0501(13)$ & $0.0859(18)$ & $-0.0109(12)$ & $0.0315(13)$ & $-0.0313(13)$ \\
N1 & $0.027(3)$ & $0.032(3)$ & $0.028(3)$ & $0.003(2)$ & $0.014(2)$ & $0.002(2)$ \\
C1 & $0.025(3)$ & $0.036(4)$ & $0.030(4)$ & $0.002(3)$ & $0.009(3)$ & $0.000(3)$ \\
C2 & $0.044(4)$ & $0.035(4)$ & $0.039(4)$ & $-0.006(3)$ & $0.023(3)$ & $-0.005(3)$ \\
C3 & $0.030(4)$ & $0.065(6)$ & $0.045(4)$ & $-0.006(4)$ & $0.014(3)$ & $-0.011(4)$ \\
C4 & $0.045(5)$ & $0.054(6)$ & $0.096(7)$ & $-0.011(4)$ & $0.043(5)$ & $-0.018(5)$ \\
C5 & $0.032(4)$ & $0.035(4)$ & $0.040(4)$ & $0.009(3)$ & $0.015(3)$ & $0.003(3)$ \\
C6 & $0.052(5)$ & $0.039(4)$ & $0.046(4)$ & $0.001(4)$ & $0.024(4)$ & $-0.007(4)$
\end{tabular}




\begin{tabular}{lllllll} 
C7 & $0.042(5)$ & $0.038(5)$ & $0.082(7)$ & $0.005(4)$ & $0.020(4)$ & $-0.012(4)$ \\
C8 & $0.063(6)$ & $0.052(6)$ & $0.116(9)$ & $-0.004(5)$ & $0.033(6)$ & $-0.034(6)$ \\
C9 & $0.024(3)$ & $0.042(4)$ & $0.025(3)$ & $0.000(3)$ & $0.010(3)$ & $0.003(3)$ \\
C10 & $0.039(4)$ & $0.043(4)$ & $0.041(4)$ & $-0.002(4)$ & $0.019(3)$ & $0.003(4)$ \\
C11 & $0.028(4)$ & $0.057(5)$ & $0.049(5)$ & $0.001(4)$ & $0.016(3)$ & $0.016(4)$ \\
C12 & $0.043(5)$ & $0.059(6)$ & $0.079(7)$ & $-0.005(4)$ & $0.027(5)$ & $0.019(5)$ \\
C13 & $0.040(4)$ & $0.043(4)$ & $0.025(3)$ & $0.004(3)$ & $0.017(3)$ & $-0.002(3)$ \\
C14 & $0.036(4)$ & $0.055(5)$ & $0.030(4)$ & $-0.003(4)$ & $0.010(3)$ & $-0.005(3)$ \\
C15 & $0.044(5)$ & $0.082(7)$ & $0.045(5)$ & $0.005(5)$ & $0.018(4)$ & $-0.005(5)$ \\
C16 & $0.056(6)$ & $0.150(12)$ & $0.035(5)$ & $0.004(7)$ & $0.009(4)$ & $-0.019(6)$ \\
C1L & $0.143(13)$ & $0.077(9)$ & $0.149(13)$ & $0.017(9)$ & $0.100(11)$ & $-0.003(9)$ \\
C11L & $0.087(2)$ & $0.168(4)$ & $0.114(3)$ & $-0.022(2)$ & $0.048(2)$ & $-0.065(3)$ \\
C12L & $0.471(12)$ & $0.237(7)$ & $0.350(10)$ & $-0.225(8)$ & $0.355(10)$ & $-0.183(7)$ \\
\hline
\end{tabular}

Geometric parameters $\left(\AA,{ }^{\circ}\right)$

\begin{tabular}{|c|c|c|c|}
\hline $\mathrm{Si} 1-\mathrm{Cl} 12$ & $2.097(3)$ & $\mathrm{C} 7-\mathrm{H} 7 \mathrm{~A}$ & 0.9900 \\
\hline Si1-Cl11 & $2.098(3)$ & $\mathrm{C} 7-\mathrm{H} 7 \mathrm{~B}$ & 0.9900 \\
\hline $\mathrm{Si} 1-\mathrm{Si} 2$ & $2.339(3)$ & $\mathrm{C} 8-\mathrm{H} 8 \mathrm{~A}$ & 0.9800 \\
\hline $\mathrm{Si} 1-\mathrm{Si}^{\mathrm{i}}$ & $2.341(4)$ & $\mathrm{C} 8-\mathrm{H} 8 \mathrm{~B}$ & 0.9800 \\
\hline $\mathrm{Si} 2-\mathrm{Cl} 21$ & $2.081(3)$ & $\mathrm{C} 8-\mathrm{H} 8 \mathrm{C}$ & 0.9800 \\
\hline $\mathrm{Si} 2-\mathrm{Cl} 22$ & $2.100(3)$ & $\mathrm{C} 9-\mathrm{C} 10$ & $1.519(10)$ \\
\hline $\mathrm{Si} 2-\mathrm{Si} 3$ & $2.347(3)$ & C9-H9A & 0.9900 \\
\hline $\mathrm{Si} 3-\mathrm{Cl} 31$ & $2.086(3)$ & С9-H9B & 0.9900 \\
\hline $\mathrm{Si} 3-\mathrm{Cl} 31^{\mathrm{i}}$ & $2.086(3)$ & $\mathrm{C} 10-\mathrm{C} 11$ & $1.509(9)$ \\
\hline $\mathrm{Si} 3-\mathrm{Si}^{2}$ & $2.347(3)$ & $\mathrm{C} 10-\mathrm{H} 10 \mathrm{~A}$ & 0.9900 \\
\hline $\mathrm{N} 1-\mathrm{C} 9$ & $1.513(8)$ & $\mathrm{C} 10-\mathrm{H} 10 \mathrm{~B}$ & 0.9900 \\
\hline $\mathrm{N} 1-\mathrm{C} 5$ & $1.515(9)$ & $\mathrm{C} 11-\mathrm{C} 12$ & $1.513(12)$ \\
\hline $\mathrm{N} 1-\mathrm{C} 1$ & $1.523(8)$ & $\mathrm{C} 11-\mathrm{H} 11 \mathrm{~A}$ & 0.9900 \\
\hline $\mathrm{N} 1-\mathrm{C} 13$ & $1.525(8)$ & $\mathrm{C} 11-\mathrm{H} 11 \mathrm{~B}$ & 0.9900 \\
\hline $\mathrm{C} 1-\mathrm{C} 2$ & $1.531(9)$ & $\mathrm{C} 12-\mathrm{H} 12 \mathrm{~A}$ & 0.9800 \\
\hline $\mathrm{C} 1-\mathrm{H} 1 \mathrm{~A}$ & 0.9900 & $\mathrm{C} 12-\mathrm{H} 12 \mathrm{~B}$ & 0.9800 \\
\hline $\mathrm{C} 1-\mathrm{H} 1 \mathrm{~B}$ & 0.9900 & $\mathrm{C} 12-\mathrm{H} 12 \mathrm{C}$ & 0.9800 \\
\hline $\mathrm{C} 2-\mathrm{C} 3$ & $1.510(10)$ & $\mathrm{C} 13-\mathrm{C} 14$ & $1.518(10)$ \\
\hline $\mathrm{C} 2-\mathrm{H} 2 \mathrm{~A}$ & 0.9900 & $\mathrm{C} 13-\mathrm{H} 13 \mathrm{~A}$ & 0.9900 \\
\hline $\mathrm{C} 2-\mathrm{H} 2 \mathrm{~B}$ & 0.9900 & $\mathrm{C} 13-\mathrm{H} 13 \mathrm{~B}$ & 0.9900 \\
\hline $\mathrm{C} 3-\mathrm{C} 4$ & $1.538(10)$ & $\mathrm{C} 14-\mathrm{C} 15$ & $1.539(10)$ \\
\hline $\mathrm{C} 3-\mathrm{H} 3 \mathrm{~A}$ & 0.9900 & $\mathrm{C} 14-\mathrm{H} 14 \mathrm{~A}$ & 0.9900 \\
\hline $\mathrm{C} 3-\mathrm{H} 3 \mathrm{~B}$ & 0.9900 & C14-H14B & 0.9900 \\
\hline $\mathrm{C} 4-\mathrm{H} 4 \mathrm{~A}$ & 0.9800 & $\mathrm{C} 15-\mathrm{C} 16$ & $1.485(12)$ \\
\hline $\mathrm{C} 4-\mathrm{H} 4 \mathrm{~B}$ & 0.9800 & C15-H15A & 0.9900 \\
\hline $\mathrm{C} 4-\mathrm{H} 4 \mathrm{C}$ & 0.9800 & $\mathrm{C} 15-\mathrm{H} 15 \mathrm{~B}$ & 0.9900 \\
\hline $\mathrm{C} 5-\mathrm{C} 6$ & $1.521(10)$ & $\mathrm{C} 16-\mathrm{H} 16 \mathrm{~A}$ & 0.9800 \\
\hline $\mathrm{C} 5-\mathrm{H} 5 \mathrm{~A}$ & 0.9900 & $\mathrm{C} 16-\mathrm{H} 16 \mathrm{~B}$ & 0.9800 \\
\hline $\mathrm{C} 5-\mathrm{H} 5 \mathrm{~B}$ & 0.9900 & $\mathrm{C} 16-\mathrm{H} 16 \mathrm{C}$ & 0.9800 \\
\hline $\mathrm{C} 6-\mathrm{C} 7$ & $1.516(11)$ & $\mathrm{C} 1 \mathrm{~L}-\mathrm{C} 12 \mathrm{~L}$ & $1.578(14)$ \\
\hline C6-H6A & 0.9900 & $\mathrm{C} 1 \mathrm{~L}-\mathrm{C} 11 \mathrm{~L}$ & $1.711(13)$ \\
\hline C6-H6B & 0.9900 & $\mathrm{C} 1 \mathrm{~L}-\mathrm{H} 1 \mathrm{~L} 1$ & 0.9900 \\
\hline
\end{tabular}




$$
\mathrm{C} 7-\mathrm{C} 8
$$

C112-Si1-Cl11

C112-Si1-Si2

C111-Si1-Si2

Cl12-Si1-Si1 ${ }^{\mathrm{i}}$

$\mathrm{C} 111-\mathrm{Si} 1-\mathrm{Si}^{\mathrm{i}}$

$\mathrm{Si} 2-\mathrm{Si} 1-\mathrm{Si}^{\mathrm{i}}$

$\mathrm{C} 121-\mathrm{Si} 2-\mathrm{Cl} 22$

$\mathrm{Cl} 21-\mathrm{Si} 2-\mathrm{Si} 1$

$\mathrm{Cl} 22-\mathrm{Si} 2-\mathrm{Si} 1$

$\mathrm{C} 121-\mathrm{Si} 2-\mathrm{Si} 3$

$\mathrm{Cl} 22-\mathrm{Si} 2-\mathrm{Si} 3$

$\mathrm{Si} 1-\mathrm{Si} 2-\mathrm{Si} 3$

$\mathrm{Cl} 31-\mathrm{Si} 3-\mathrm{Cl} 31^{\mathrm{i}}$

$\mathrm{Cl} 31-\mathrm{Si} 3-\mathrm{Si} 2$

$\mathrm{Cl} 31^{\mathrm{i}}-\mathrm{Si} 3-\mathrm{Si} 2$

$\mathrm{Cl} 31-\mathrm{Si} 3-\mathrm{Si}^{2}$

$\mathrm{Cl} 31^{\mathrm{i}}-\mathrm{Si} 3-\mathrm{Si} 2^{\mathrm{i}}$

$\mathrm{Si} 2-\mathrm{Si} 3-\mathrm{Si}_{2}^{\mathrm{i}}$

C9-N1-C5

C9-N1-C1

C5-N1-C1

$\mathrm{C} 9-\mathrm{N} 1-\mathrm{C} 13$

C5-N1-C13

$\mathrm{C} 1-\mathrm{N} 1-\mathrm{C} 13$

$\mathrm{N} 1-\mathrm{C} 1-\mathrm{C} 2$

$\mathrm{N} 1-\mathrm{C} 1-\mathrm{H} 1 \mathrm{~A}$

$\mathrm{C} 2-\mathrm{C} 1-\mathrm{H} 1 \mathrm{~A}$

$\mathrm{N} 1-\mathrm{C} 1-\mathrm{H} 1 \mathrm{~B}$

$\mathrm{C} 2-\mathrm{C} 1-\mathrm{H} 1 \mathrm{~B}$

H1A-C1-H1B

$\mathrm{C} 3-\mathrm{C} 2-\mathrm{C} 1$

$\mathrm{C} 3-\mathrm{C} 2-\mathrm{H} 2 \mathrm{~A}$

$\mathrm{C} 1-\mathrm{C} 2-\mathrm{H} 2 \mathrm{~A}$

$\mathrm{C} 3-\mathrm{C} 2-\mathrm{H} 2 \mathrm{~B}$

$\mathrm{C} 1-\mathrm{C} 2-\mathrm{H} 2 \mathrm{~B}$

$\mathrm{H} 2 \mathrm{~A}-\mathrm{C} 2-\mathrm{H} 2 \mathrm{~B}$

$\mathrm{C} 2-\mathrm{C} 3-\mathrm{C} 4$

$\mathrm{C} 2-\mathrm{C} 3-\mathrm{H} 3 \mathrm{~A}$

$\mathrm{C} 4-\mathrm{C} 3-\mathrm{H} 3 \mathrm{~A}$

$\mathrm{C} 2-\mathrm{C} 3-\mathrm{H} 3 \mathrm{~B}$

$\mathrm{C} 4-\mathrm{C} 3-\mathrm{H} 3 \mathrm{~B}$

$\mathrm{H} 3 \mathrm{~A}-\mathrm{C} 3-\mathrm{H} 3 \mathrm{~B}$

$\mathrm{C} 3-\mathrm{C} 4-\mathrm{H} 4 \mathrm{~A}$

$\mathrm{C} 3-\mathrm{C} 4-\mathrm{H} 4 \mathrm{~B}$

$\mathrm{H} 4 \mathrm{~A}-\mathrm{C} 4-\mathrm{H} 4 \mathrm{~B}$

$\mathrm{C} 3-\mathrm{C} 4-\mathrm{H} 4 \mathrm{C}$

\section{$1.522(13)$}

99.57 (12)

$111.06(11)$

$114.22(11)$

$112.32(12)$

$111.28(12)$

$108.28(6)$

$99.76(11)$

$114.16(12)$

$110.71(12)$

$111.61(12)$

$113.15(11)$

107.47 (10)

$99.6(2)$

111.65 (9)

$112.64(9)$

$112.64(9)$

$111.65(9)$

$108.50(16)$

$108.1(5)$

$109.8(5)$

$111.2(5)$

$110.9(5)$

$108.8(5)$

$108.0(5)$

$116.1(6)$

108.3

108.3

108.3

108.3

107.4

$110.1(6)$

109.6

109.6

109.6

109.6

108.1

$112.1(7)$

109.2

109.2

109.2

109.2

107.9

109.5

109.5

109.5

109.5

$$
\text { C1L-H1L2 }
$$

$\mathrm{C} 6-\mathrm{C} 7-\mathrm{H} 7 \mathrm{~B}$

$\mathrm{C} 8-\mathrm{C} 7-\mathrm{H} 7 \mathrm{~B}$

$\mathrm{H} 7 \mathrm{~A}-\mathrm{C} 7-\mathrm{H} 7 \mathrm{~B}$

C7- 8 - $-\mathrm{H} 8 \mathrm{~A}$

$\mathrm{C} 7-\mathrm{C} 8-\mathrm{H} 8 \mathrm{~B}$

$\mathrm{H} 8 \mathrm{~A}-\mathrm{C} 8-\mathrm{H} 8 \mathrm{~B}$

C7-C $8-\mathrm{H} 8 \mathrm{C}$

$\mathrm{H} 8 \mathrm{~A}-\mathrm{C} 8-\mathrm{H} 8 \mathrm{C}$

$\mathrm{H} 8 \mathrm{~B}-\mathrm{C} 8-\mathrm{H} 8 \mathrm{C}$

$\mathrm{N} 1-\mathrm{C} 9-\mathrm{C} 10$

$\mathrm{N} 1-\mathrm{C} 9-\mathrm{H} 9 \mathrm{~A}$

$\mathrm{C} 10-\mathrm{C} 9-\mathrm{H} 9 \mathrm{~A}$

N1-C9-H9B

$\mathrm{C} 10-\mathrm{C} 9-\mathrm{H} 9 \mathrm{~B}$

H9A-C9-H9B

$\mathrm{C} 11-\mathrm{C} 10-\mathrm{C} 9$

$\mathrm{C} 11-\mathrm{C} 10-\mathrm{H} 10 \mathrm{~A}$

C9- $\mathrm{C} 10-\mathrm{H} 10 \mathrm{~A}$

C11-C10-H10B

C9- $\mathrm{C} 10-\mathrm{H} 10 \mathrm{~B}$

$\mathrm{H} 10 \mathrm{~A}-\mathrm{C} 10-\mathrm{H} 10 \mathrm{~B}$

$\mathrm{C} 10-\mathrm{C} 11-\mathrm{C} 12$

C10-C11-H11A

C12-C11-H11A

$\mathrm{C} 10-\mathrm{C} 11-\mathrm{H} 11 \mathrm{~B}$

C12-C11-H11B

H11A-C11-H11B

$\mathrm{C} 11-\mathrm{C} 12-\mathrm{H} 12 \mathrm{~A}$

C11-C12-H12B

$\mathrm{H} 12 \mathrm{~A}-\mathrm{C} 12-\mathrm{H} 12 \mathrm{~B}$

$\mathrm{C} 11-\mathrm{C} 12-\mathrm{H} 12 \mathrm{C}$

$\mathrm{H} 12 \mathrm{~A}-\mathrm{C} 12-\mathrm{H} 12 \mathrm{C}$

$\mathrm{H} 12 \mathrm{~B}-\mathrm{C} 12-\mathrm{H} 12 \mathrm{C}$

$\mathrm{C} 14-\mathrm{C} 13-\mathrm{N} 1$

C14-C13-H13A

$\mathrm{N} 1-\mathrm{C} 13-\mathrm{H} 13 \mathrm{~A}$

C14-C13-H13B

N1-C13-H13B

H13A-C13-H13B

C13-C14-C15

C13-C14-H14A

C15-C14-H14A

C13-C14-H14B

C15-C14-H14B

$\mathrm{H} 14 \mathrm{~A}-\mathrm{C} 14-\mathrm{H} 14 \mathrm{~B}$

C16-C15-C14
0.9900

109.2

109.2

107.9

109.5

109.5

109.5

109.5

109.5

109.5

$116.6(6)$

108.1

108.1

108.1

108.1

107.3

$110.0(6)$

109.7

109.7

109.7

109.7

108.2

112.6 (7)

109.1

109.1

109.1

109.1

107.8

109.5

109.5

109.5

109.5

109.5

109.5

116.9 (6)

108.1

108.1

108.1

108.1

107.3

108.9 (6)

109.9

109.9

109.9

109.9

108.3

112.5 (7) 


\begin{tabular}{|c|c|c|c|}
\hline $\mathrm{H} 4 \mathrm{~A}-\mathrm{C} 4-\mathrm{H} 4 \mathrm{C}$ & 109.5 & $\mathrm{C} 16-\mathrm{C} 15-\mathrm{H} 15 \mathrm{~A}$ & 109.1 \\
\hline $\mathrm{H} 4 \mathrm{~B}-\mathrm{C} 4-\mathrm{H} 4 \mathrm{C}$ & 109.5 & $\mathrm{C} 14-\mathrm{C} 15-\mathrm{H} 15 \mathrm{~A}$ & 109.1 \\
\hline $\mathrm{N} 1-\mathrm{C} 5-\mathrm{C} 6$ & $115.6(6)$ & $\mathrm{C} 16-\mathrm{C} 15-\mathrm{H} 15 \mathrm{~B}$ & 109.1 \\
\hline $\mathrm{N} 1-\mathrm{C} 5-\mathrm{H} 5 \mathrm{~A}$ & 108.4 & $\mathrm{C} 14-\mathrm{C} 15-\mathrm{H} 15 \mathrm{~B}$ & 109.1 \\
\hline $\mathrm{C} 6-\mathrm{C} 5-\mathrm{H} 5 \mathrm{~A}$ & 108.4 & $\mathrm{H} 15 \mathrm{~A}-\mathrm{C} 15-\mathrm{H} 15 \mathrm{~B}$ & 107.8 \\
\hline $\mathrm{N} 1-\mathrm{C} 5-\mathrm{H} 5 \mathrm{~B}$ & 108.4 & $\mathrm{C} 15-\mathrm{C} 16-\mathrm{H} 16 \mathrm{~A}$ & 109.5 \\
\hline $\mathrm{C} 6-\mathrm{C} 5-\mathrm{H} 5 \mathrm{~B}$ & 108.4 & $\mathrm{C} 15-\mathrm{C} 16-\mathrm{H} 16 \mathrm{~B}$ & 109.5 \\
\hline $\mathrm{H} 5 \mathrm{~A}-\mathrm{C} 5-\mathrm{H} 5 \mathrm{~B}$ & 107.4 & $\mathrm{H} 16 \mathrm{~A}-\mathrm{C} 16-\mathrm{H} 16 \mathrm{~B}$ & 109.5 \\
\hline $\mathrm{C} 7-\mathrm{C} 6-\mathrm{C} 5$ & $110.1(7)$ & $\mathrm{C} 15-\mathrm{C} 16-\mathrm{H} 16 \mathrm{C}$ & 109.5 \\
\hline $\mathrm{C} 7-\mathrm{C} 6-\mathrm{H} 6 \mathrm{~A}$ & 109.6 & $\mathrm{H} 16 \mathrm{~A}-\mathrm{C} 16-\mathrm{H} 16 \mathrm{C}$ & 109.5 \\
\hline $\mathrm{C} 5-\mathrm{C} 6-\mathrm{H} 6 \mathrm{~A}$ & 109.6 & $\mathrm{H} 16 \mathrm{~B}-\mathrm{C} 16-\mathrm{H} 16 \mathrm{C}$ & 109.5 \\
\hline $\mathrm{C} 7-\mathrm{C} 6-\mathrm{H} 6 \mathrm{~B}$ & 109.6 & $\mathrm{Cl} 2 \mathrm{~L}-\mathrm{C} 1 \mathrm{~L}-\mathrm{Cl} 1 \mathrm{~L}$ & $116.2(8)$ \\
\hline $\mathrm{C} 5-\mathrm{C} 6-\mathrm{H} 6 \mathrm{~B}$ & 109.6 & $\mathrm{Cl} 2 \mathrm{~L}-\mathrm{C} 1 \mathrm{~L}-\mathrm{H} 1 \mathrm{~L} 1$ & 108.2 \\
\hline $\mathrm{H} 6 \mathrm{~A}-\mathrm{C} 6-\mathrm{H} 6 \mathrm{~B}$ & 108.2 & $\mathrm{C} 11 \mathrm{~L}-\mathrm{C} 1 \mathrm{~L}-\mathrm{H} 1 \mathrm{~L} 1$ & 108.2 \\
\hline $\mathrm{C} 6-\mathrm{C} 7-\mathrm{C} 8$ & $112.3(8)$ & $\mathrm{Cl} 2 \mathrm{~L}-\mathrm{C} 1 \mathrm{~L}-\mathrm{H} 1 \mathrm{~L} 2$ & 108.2 \\
\hline $\mathrm{C} 6-\mathrm{C} 7-\mathrm{H} 7 \mathrm{~A}$ & 109.2 & $\mathrm{C} 11 \mathrm{~L}-\mathrm{C} 1 \mathrm{~L}-\mathrm{H} 1 \mathrm{~L} 2$ & 108.2 \\
\hline $\mathrm{C} 8-\mathrm{C} 7-\mathrm{H} 7 \mathrm{~A}$ & 109.2 & $\mathrm{H} 1 \mathrm{~L} 1-\mathrm{C} 1 \mathrm{~L}-\mathrm{H} 1 \mathrm{~L} 2$ & 107.4 \\
\hline $\mathrm{C} 9-\mathrm{N} 1-\mathrm{C} 1-\mathrm{C} 2$ & $-174.3(6)$ & $\mathrm{C} 5-\mathrm{N} 1-\mathrm{C} 9-\mathrm{C} 10$ & $175.4(6)$ \\
\hline $\mathrm{C} 5-\mathrm{N} 1-\mathrm{C} 1-\mathrm{C} 2$ & $-54.8(7)$ & $\mathrm{C} 1-\mathrm{N} 1-\mathrm{C} 9-\mathrm{C} 10$ & $-63.2(7)$ \\
\hline $\mathrm{C} 13-\mathrm{N} 1-\mathrm{C} 1-\mathrm{C} 2$ & $64.6(7)$ & $\mathrm{C} 13-\mathrm{N} 1-\mathrm{C} 9-\mathrm{C} 10$ & $56.1(8)$ \\
\hline $\mathrm{N} 1-\mathrm{C} 1-\mathrm{C} 2-\mathrm{C} 3$ & $-179.8(6)$ & $\mathrm{N} 1-\mathrm{C} 9-\mathrm{C} 10-\mathrm{C} 11$ & $-175.2(6)$ \\
\hline $\mathrm{C} 1-\mathrm{C} 2-\mathrm{C} 3-\mathrm{C} 4$ & $172.9(7)$ & $\mathrm{C} 9-\mathrm{C} 10-\mathrm{C} 11-\mathrm{C} 12$ & $-176.1(7)$ \\
\hline $\mathrm{C} 9-\mathrm{N} 1-\mathrm{C} 5-\mathrm{C} 6$ & $68.4(7)$ & $\mathrm{C} 9-\mathrm{N} 1-\mathrm{C} 13-\mathrm{C} 14$ & $55.2(8)$ \\
\hline $\mathrm{C} 1-\mathrm{N} 1-\mathrm{C} 5-\mathrm{C} 6$ & $-52.2(7)$ & $\mathrm{C} 5-\mathrm{N} 1-\mathrm{C} 13-\mathrm{C} 14$ & $-63.6(8)$ \\
\hline $\mathrm{C} 13-\mathrm{N} 1-\mathrm{C} 5-\mathrm{C} 6$ & $-171.0(6)$ & $\mathrm{C} 1-\mathrm{N} 1-\mathrm{C} 13-\mathrm{C} 14$ & $175.6(6)$ \\
\hline $\mathrm{N} 1-\mathrm{C} 5-\mathrm{C} 6-\mathrm{C} 7$ & $-179.9(6)$ & $\mathrm{N} 1-\mathrm{C} 13-\mathrm{C} 14-\mathrm{C} 15$ & $176.8(7)$ \\
\hline $\mathrm{C} 5-\mathrm{C} 6-\mathrm{C} 7-\mathrm{C} 8$ & $-177.3(7)$ & $\mathrm{C} 13-\mathrm{C} 14-\mathrm{C} 15-\mathrm{C} 16$ & $-175.4(9)$ \\
\hline
\end{tabular}

Symmetry code: (i) $-x+1, y,-z+3 / 2$.

Hydrogen-bond geometry $\left(\AA,{ }^{\circ}\right)$

\begin{tabular}{lllll}
\hline$D-\mathrm{H} \cdots A$ & $D-\mathrm{H}$ & $\mathrm{H} \cdots A$ & $D \cdots A$ & $D-\mathrm{H} \cdots A$ \\
\hline $\mathrm{C} 1-\mathrm{H} 1 B \cdots \mathrm{C} 11$ & 0.99 & 2.88 & $3.686(7)$ & 139 \\
$\mathrm{C} 2-\mathrm{H} 2 A \cdots \mathrm{Cl} 31^{\mathrm{ii}}$ & 0.99 & 2.89 & $3.596(8)$ & 129 \\
$\mathrm{C} 5-\mathrm{H} 5 A \cdots \mathrm{Cl} 22^{\mathrm{iii}}$ & 0.99 & 2.99 & $3.945(7)$ & 163 \\
$\mathrm{C} 9-\mathrm{H} 9 B \cdots \mathrm{C} 11$ & 0.99 & 2.91 & $3.652(7)$ & 132 \\
$\mathrm{C} 1 L-\mathrm{H} 1 L 1 \cdots \mathrm{C} 112^{\mathrm{iii}}$ & 0.99 & 2.96 & $3.528(13)$ & 119 \\
\hline
\end{tabular}

Symmetry codes: (ii) $-x+1,-y+1,-z+1$; (iii) $x-1 / 2,-y+3 / 2, z-1 / 2$.

Bis(tetraethylammonium) dichloride decachlorocyclopentasilane dichloromethane disolvate (II)

Crystal data

$2 \mathrm{C}_{8} \mathrm{H}_{20} \mathrm{~N}^{+} \cdot 2 \mathrm{Cl}^{-} \cdot \mathrm{Si}_{5} \mathrm{Cl}_{10} \cdot 2 \mathrm{CH}_{2} \mathrm{Cl}_{2}$

$M_{r}=996.20$

Triclinic, $P \overline{1}$

$a=10.3596(4) \AA$

$b=13.9612(5) \AA$

$$
\begin{aligned}
& c=16.0205(6) \AA \\
& \alpha=89.959(3)^{\circ} \\
& \beta=72.484(3)^{\circ} \\
& \gamma=79.534(3)^{\circ} \\
& V=2169.29(15) \AA^{3}
\end{aligned}
$$




\section{$Z=2$}

$F(000)=1016$

$D_{\mathrm{x}}=1.525 \mathrm{Mg} \mathrm{m}^{-3}$

Mo $K \alpha$ radiation, $\lambda=0.71073 \AA$

Cell parameters from 153722 reflections

\section{Data collection}

Stoe IPDS II two-circle diffractometer

$\omega$ scans

Absorption correction: multi-scan

(X-AREA; Stoe \& Cie, 2001)

$T_{\min }=0.408, T_{\max }=1.000$

62962 measured reflections

\section{Refinement}

Refinement on $F^{2}$

Least-squares matrix: full

$R\left[F^{2}>2 \sigma\left(F^{2}\right)\right]=0.048$

$w R\left(F^{2}\right)=0.131$

$S=1.08$

13044 reflections

409 parameters

0 restraints $\theta=3.3-30.8^{\circ}$

$\mu=1.17 \mathrm{~mm}^{-1}$

$T=173 \mathrm{~K}$

Block, colourless

$0.23 \times 0.23 \times 0.20 \mathrm{~mm}$

13044 independent reflections

11976 reflections with $I>2 \sigma(I)$

$R_{\text {int }}=0.043$

$\theta_{\text {max }}=30.5^{\circ}, \theta_{\text {min }}=3.4^{\circ}$

$h=-14 \rightarrow 14$

$k=-19 \rightarrow 19$

$l=-22 \rightarrow 22$

Hydrogen site location: inferred from neighbouring sites

$\mathrm{H}$-atom parameters constrained

$w=1 /\left[\sigma^{2}\left(F_{\mathrm{o}}^{2}\right)+(0.0683 P)^{2}+1.6519 P\right]$

where $P=\left(F_{\mathrm{o}}^{2}+2 F_{\mathrm{c}}^{2}\right) / 3$

$(\Delta / \sigma)_{\max }=0.001$

$\Delta \rho_{\max }=0.87 \mathrm{e} \AA^{-3}$

$\Delta \rho_{\min }=-0.84$ e $\AA^{-3}$

Special details

Geometry. All esds (except the esd in the dihedral angle between two 1.s. planes) are estimated using the full covariance matrix. The cell esds are taken into account individually in the estimation of esds in distances, angles and torsion angles; correlations between esds in cell parameters are only used when they are defined by crystal symmetry. An approximate (isotropic) treatment of cell esds is used for estimating esds involving 1.s. planes.

Fractional atomic coordinates and isotropic or equivalent isotropic displacement parameters $\left(\AA^{2}\right)$

\begin{tabular}{|c|c|c|c|c|c|}
\hline & $x$ & $y$ & $z$ & $U_{\text {iso }} * / U_{\text {eq }}$ & Occ. $(<1)$ \\
\hline $\mathrm{Cl1}$ & $0.68362(5)$ & $0.71247(4)$ & $0.37887(3)$ & $0.03357(10)$ & \\
\hline $\mathrm{Cl} 2$ & $0.55394(6)$ & $0.71721(4)$ & $0.14756(3)$ & $0.03425(11)$ & \\
\hline Sil & $0.79603(6)$ & $0.63165(4)$ & $0.19539(4)$ & $0.03316(12)$ & \\
\hline $\mathrm{Si} 2$ & $0.74595(6)$ & $0.80223(4)$ & $0.20699(4)$ & 0.02939 (11) & \\
\hline $\mathrm{Si} 3$ & $0.51729(5)$ & $0.85241(4)$ & $0.29623(3)$ & $0.02570(10)$ & \\
\hline $\mathrm{Si} 4$ & $0.42527(5)$ & $0.71172(4)$ & 0.33588 & $0.02431(10)$ & \\
\hline $\mathrm{Si} 5$ & $0.59853(6)$ & $0.57465(4)$ & $0.27696(4)$ & $0.02724(11)$ & \\
\hline Cl11 & $0.97197(6)$ & $0.57714(6)$ & $0.23162(6)$ & $0.0628(2)$ & \\
\hline $\mathrm{Cl12}$ & $0.86579(9)$ & $0.57414(5)$ & $0.06642(4)$ & $0.0632(2)$ & \\
\hline $\mathrm{Cl} 21$ & $0.88627(7)$ & $0.86092(6)$ & $0.25209(5)$ & $0.05179(16)$ & \\
\hline $\mathrm{Cl} 22$ & $0.78501(6)$ & $0.86177(4)$ & $0.08273(4)$ & $0.04303(13)$ & \\
\hline $\mathrm{Cl} 31$ & $0.49581(7)$ & 0.94164 (4) & $0.40573(4)$ & 0.04399 (13) & \\
\hline $\mathrm{Cl} 32$ & $0.39997(6)$ & $0.94686(4)$ & $0.23306(4)$ & $0.04020(12)$ & \\
\hline $\mathrm{Cl} 41$ & $0.33929(5)$ & $0.71094(4)$ & $0.47227(3)$ & $0.03436(10)$ & \\
\hline $\mathrm{C} 142$ & $0.24659(5)$ & $0.71209(4)$ & $0.29964(4)$ & $0.03703(11)$ & \\
\hline $\mathrm{Cl} 51$ & $0.63586(6)$ & $0.47736(4)$ & $0.36927(4)$ & $0.04057(12)$ & \\
\hline $\mathrm{Cl} 52$ & $0.53223(8)$ & $0.48498(4)$ & $0.20041(4)$ & $0.04831(15)$ & \\
\hline
\end{tabular}




\begin{tabular}{|c|c|c|c|c|c|}
\hline N1 & $0.27041(18)$ & $0.79721(13)$ & $-0.00541(11)$ & $0.0299(3)$ & \\
\hline $\mathrm{C} 1$ & $0.2204(4)$ & $0.7299(2)$ & $0.06657(18)$ & $0.0551(7)$ & \\
\hline $\mathrm{H} 1 \mathrm{~A}$ & 0.1238 & 0.7262 & 0.0717 & $0.066^{*}$ & \\
\hline H1B & 0.2206 & 0.7590 & 0.1228 & $0.066^{*}$ & \\
\hline $\mathrm{C} 2$ & $0.3050(4)$ & 0.62647 (19) & $0.0530(2)$ & $0.0551(7)$ & \\
\hline $\mathrm{H} 2 \mathrm{~A}$ & 0.2656 & 0.5882 & 0.1024 & $0.083^{*}$ & \\
\hline $\mathrm{H} 2 \mathrm{~B}$ & 0.3034 & 0.5959 & -0.0016 & $0.083^{*}$ & \\
\hline $\mathrm{H} 2 \mathrm{C}$ & 0.4004 & 0.6288 & 0.0497 & $0.083^{*}$ & \\
\hline $\mathrm{C} 3$ & $0.2867(3)$ & $0.7533(2)$ & $-0.09498(16)$ & $0.0438(5)$ & \\
\hline $\mathrm{H} 3 \mathrm{~A}$ & 0.3641 & 0.6966 & -0.1089 & $0.053^{*}$ & \\
\hline H3B & 0.3126 & 0.8021 & -0.1387 & $0.053^{*}$ & \\
\hline $\mathrm{C} 4$ & $0.1610(4)$ & $0.7201(3)$ & $-0.1052(3)$ & $0.0648(9)$ & \\
\hline $\mathrm{H} 4 \mathrm{~A}$ & 0.1816 & 0.6928 & -0.1651 & $0.097 *$ & \\
\hline H4B & 0.1357 & 0.6701 & -0.0636 & $0.097 *$ & \\
\hline $\mathrm{H} 4 \mathrm{C}$ & 0.0842 & 0.7759 & -0.0934 & $0.097 *$ & \\
\hline $\mathrm{C} 5$ & $0.1640(3)$ & 0.89268 (19) & $0.0117(2)$ & $0.0470(6)$ & \\
\hline $\mathrm{H} 5 \mathrm{~A}$ & 0.1863 & 0.9314 & -0.0407 & $0.056^{*}$ & \\
\hline H5B & 0.0721 & 0.8767 & 0.0192 & $0.056^{*}$ & \\
\hline C6 & $0.1555(3)$ & $0.9554(2)$ & $0.0910(2)$ & $0.0545(7)$ & \\
\hline H6A & 0.0852 & 1.0145 & 0.0967 & $0.082^{*}$ & \\
\hline H6B & 0.1306 & 0.9187 & 0.1438 & $0.082^{*}$ & \\
\hline $\mathrm{H} 6 \mathrm{C}$ & 0.2451 & 0.9736 & 0.0837 & $0.082 *$ & \\
\hline $\mathrm{C} 7$ & $0.4085(3)$ & $0.81784(18)$ & $-0.00349(18)$ & $0.0412(5)$ & \\
\hline H7A & 0.3980 & 0.8424 & 0.0566 & $0.049^{*}$ & \\
\hline H7B & 0.4756 & 0.7554 & -0.0155 & $0.049^{*}$ & \\
\hline $\mathrm{C} 8$ & $0.4683(3)$ & $0.8892(2)$ & $-0.0662(2)$ & $0.0517(6)$ & \\
\hline H8A & 0.5568 & 0.8970 & -0.0594 & $0.078^{*}$ & \\
\hline H8B & 0.4826 & 0.8651 & -0.1264 & $0.078^{*}$ & \\
\hline $\mathrm{H} 8 \mathrm{C}$ & 0.4048 & 0.9523 & -0.0540 & $0.078^{*}$ & \\
\hline N2 & 0.0000 & 0.5000 & 0.5000 & $0.0260(4)$ & \\
\hline $\mathrm{C} 21$ & -0.0005 & $0.6091(3)$ & $0.5117(3)$ & $0.0331(8)$ & 0.5 \\
\hline $\mathrm{H} 21 \mathrm{~A}$ & -0.0855 & 0.6483 & 0.5040 & $0.040^{*}$ & 0.5 \\
\hline $\mathrm{H} 21 \mathrm{~B}$ & 0.0800 & 0.6270 & 0.4675 & $0.040^{*}$ & 0.5 \\
\hline $\mathrm{C} 21^{\prime}$ & $0.0128(5)$ & $0.5139(3)$ & $0.5909(3)$ & $0.0350(8)$ & 0.5 \\
\hline $\mathrm{H} 21 \mathrm{C}$ & 0.1013 & 0.4755 & 0.5941 & $0.042^{*}$ & 0.5 \\
\hline H21D & -0.0633 & 0.4912 & 0.6352 & $0.042^{*}$ & 0.5 \\
\hline $\mathrm{C} 22$ & $0.0063(3)$ & $0.6300(3)$ & $0.6105(2)$ & $0.0638(9)$ & \\
\hline $\mathrm{H} 22 \mathrm{~A}$ & 0.0061 & 0.6994 & 0.6197 & $0.096^{*}$ & 0.5 \\
\hline $\mathrm{H} 22 \mathrm{~B}$ & -0.0740 & 0.6122 & 0.6536 & $0.096^{*}$ & 0.5 \\
\hline $\mathrm{H} 22 \mathrm{C}$ & 0.0908 & 0.5910 & 0.6173 & $0.096^{*}$ & 0.5 \\
\hline $\mathrm{H} 22 \mathrm{D}$ & 0.0144 & 0.6403 & 0.6690 & $0.096^{*}$ & 0.5 \\
\hline $\mathrm{H} 22 \mathrm{E}$ & 0.0823 & 0.6518 & 0.5665 & $0.096^{*}$ & 0.5 \\
\hline $\mathrm{H} 22 \mathrm{~F}$ & -0.0818 & 0.6675 & 0.6075 & $0.096^{*}$ & 0.5 \\
\hline $\mathrm{C} 23$ & 0.1319 (4) & $0.4394(3)$ & 0.5085 & $0.0294(7)$ & 0.5 \\
\hline $\mathrm{H} 23 \mathrm{~A}$ & 0.1285 & 0.3693 & 0.5040 & $0.035^{*}$ & 0.5 \\
\hline $\mathrm{H} 23 \mathrm{~B}$ & 0.1411 & 0.4544 & 0.5666 & $0.035^{*}$ & 0.5 \\
\hline C23' & $0.1182(4)$ & $0.5289(3)$ & 0.4300 & $0.0321(7)$ & 0.5 \\
\hline $\mathrm{H} 23 \mathrm{C}$ & 0.1196 & 0.5986 & 0.4398 & $0.039 *$ & 0.5 \\
\hline
\end{tabular}




\begin{tabular}{|c|c|c|c|c|c|}
\hline H23D & 0.1063 & 0.5197 & 0.3717 & $0.039 *$ & 0.5 \\
\hline $\mathrm{C} 24$ & $0.2624(2)$ & $0.4624(2)$ & $0.4327(2)$ & $0.0533(7)$ & \\
\hline $\mathrm{H} 24 \mathrm{~A}$ & 0.3471 & 0.4228 & 0.4389 & $0.080^{*}$ & 0.5 \\
\hline H24B & 0.2538 & 0.4467 & 0.3753 & $0.080^{*}$ & 0.5 \\
\hline $\mathrm{H} 24 \mathrm{C}$ & 0.2662 & 0.5317 & 0.4377 & $0.080 *$ & 0.5 \\
\hline $\mathrm{H} 24 \mathrm{D}$ & 0.3391 & 0.4813 & 0.3871 & $0.080^{*}$ & 0.5 \\
\hline $\mathrm{H} 24 \mathrm{E}$ & 0.2743 & 0.4723 & 0.4903 & $0.080 *$ & 0.5 \\
\hline $\mathrm{H} 24 \mathrm{~F}$ & 0.2610 & 0.3935 & 0.4224 & $0.080^{*}$ & 0.5 \\
\hline N3 & 0.0000 & 1.0000 & 0.5000 & $0.0324(5)$ & \\
\hline C31 & $-0.0124(7)$ & $1.0998(4)$ & $0.5376(4)$ & $0.0522(13)$ & 0.5 \\
\hline H31A & -0.0599 & 1.1032 & 0.6015 & $0.063^{*}$ & 0.5 \\
\hline H31B & -0.0684 & 1.1477 & 0.5106 & $0.063^{*}$ & 0.5 \\
\hline $\mathrm{C} 31^{\prime}$ & $0.1452(5)$ & $1.0189(5)$ & $0.4881(4)$ & $0.0509(13)$ & 0.5 \\
\hline $\mathrm{H} 31 \mathrm{C}$ & 0.2003 & 1.0076 & 0.4255 & $0.061 *$ & 0.5 \\
\hline H31D & 0.1910 & 0.9729 & 0.5221 & $0.061^{*}$ & 0.5 \\
\hline C32 & $0.1392(6)$ & $1.1271(4)$ & $0.5199(3)$ & $0.0956(17)$ & \\
\hline $\mathrm{H} 32 \mathrm{~A}$ & 0.1294 & 1.1930 & 0.5450 & $0.143^{*}$ & 0.5 \\
\hline H32B & 0.1941 & 1.0803 & 0.5474 & $0.143^{*}$ & 0.5 \\
\hline $\mathrm{H} 32 \mathrm{C}$ & 0.1855 & 1.1246 & 0.4566 & $0.143^{*}$ & 0.5 \\
\hline H32D & 0.2330 & 1.1379 & 0.5118 & $0.143 *$ & 0.5 \\
\hline $\mathrm{H} 32 \mathrm{E}$ & 0.0951 & 1.1727 & 0.4856 & $0.143^{*}$ & 0.5 \\
\hline $\mathrm{H} 32 \mathrm{~F}$ & 0.0857 & 1.1380 & 0.5821 & $0.143 *$ & 0.5 \\
\hline C33 & $-0.0905(5)$ & $1.0176(4)$ & $0.5927(3)$ & $0.0412(10)$ & 0.5 \\
\hline H33A & -0.1850 & 1.0092 & 0.5967 & $0.049 *$ & 0.5 \\
\hline H33B & -0.0952 & 1.0851 & 0.6140 & $0.049 *$ & 0.5 \\
\hline C33' & $0.0691(5)$ & $0.9243(4)$ & 0.5529 & $0.0459(11)$ & 0.5 \\
\hline $\mathrm{H} 33 \mathrm{C}$ & 0.1629 & 0.9350 & 0.5479 & $0.055^{*}$ & 0.5 \\
\hline H33D & 0.0765 & 0.8572 & 0.5296 & $0.055^{*}$ & 0.5 \\
\hline C34 & -0.0250 & $0.9384(3)$ & $0.6531(2)$ & $0.0724(11)$ & \\
\hline H34A & -0.0833 & 0.9490 & 0.7144 & $0.109 *$ & 0.5 \\
\hline H34B & -0.0212 & 0.8719 & 0.6318 & $0.109 *$ & 0.5 \\
\hline $\mathrm{H} 34 \mathrm{C}$ & 0.0683 & 0.9475 & 0.6490 & $0.109 *$ & 0.5 \\
\hline H34D & 0.0162 & 0.8916 & 0.6879 & $0.109 *$ & 0.5 \\
\hline H34E & -0.0314 & 1.0050 & 0.6756 & $0.109 *$ & 0.5 \\
\hline $\mathrm{H} 34 \mathrm{~F}$ & -0.1175 & 0.9274 & 0.6574 & $0.109 *$ & 0.5 \\
\hline $\mathrm{C} 1 \mathrm{~L}$ & 0.1880 & $0.3162(2)$ & $0.1144(2)$ & $0.0565(7)$ & \\
\hline H1L1 & 0.2330 & 0.2960 & 0.0516 & $0.068 *$ & \\
\hline H1L2 & 0.0997 & 0.3611 & 0.1200 & $0.068^{*}$ & \\
\hline $\mathrm{Cl1A}$ & $0.29537(8)$ & $0.37721(5)$ & $0.15334(6)$ & $0.05663(17)$ & \\
\hline C11B & $0.15522(9)$ & $0.21224(6)$ & $0.17452(6)$ & $0.05883(18)$ & \\
\hline $\mathrm{C} 2 \mathrm{~L}$ & $0.4865(4)$ & $0.7900(3)$ & $0.6208(2)$ & $0.0642(8)$ & \\
\hline H2L1 & 0.5201 & 0.8228 & 0.5657 & $0.077^{*}$ & \\
\hline H2L2 & 0.4831 & 0.7222 & 0.6050 & $0.077 *$ & \\
\hline $\mathrm{Cl} 2 \mathrm{~A}$ & $0.60192(10)$ & $0.78831(6)$ & $0.68194(6)$ & $0.0634(2)$ & \\
\hline $\mathrm{C} 12 \mathrm{~B}$ & $0.32076(10)$ & $0.85079(10)$ & $0.67916(6)$ & 0.0828 & \\
\hline
\end{tabular}


Atomic displacement parameters $\left(\AA^{2}\right)$

\begin{tabular}{|c|c|c|c|c|c|c|}
\hline & $U^{11}$ & $U^{22}$ & $U^{33}$ & $U^{12}$ & $U^{13}$ & $U^{23}$ \\
\hline $\mathrm{C} 11$ & $0.0361(2)$ & $0.0429(3)$ & $0.0274(2)$ & -0.01369 (19) & $-0.01464(17)$ & $0.00581(17)$ \\
\hline $\mathrm{Cl} 2$ & $0.0424(3)$ & $0.0357(2)$ & $0.0271(2)$ & -0.00201 (19) & $-0.01742(18)$ & $-0.00062(16)$ \\
\hline Sil & $0.0284(2)$ & $0.0311(3)$ & $0.0307(3)$ & $0.0042(2)$ & $-0.0012(2)$ & $0.0051(2)$ \\
\hline $\mathrm{Si} 2$ & $0.0258(2)$ & $0.0307(3)$ & $0.0279(2)$ & -0.00452 (19) & $-0.00314(19)$ & 0.00405 (19) \\
\hline $\mathrm{Si} 3$ & $0.0266(2)$ & $0.0229(2)$ & $0.0245(2)$ & $-0.00328(17)$ & $-0.00405(18)$ & $-0.00117(17)$ \\
\hline $\mathrm{Si} 4$ & $0.0239(2)$ & $0.0234(2)$ & $0.0246(2)$ & $-0.00303(17)$ & $-0.00662(17)$ & $-0.00054(16)$ \\
\hline $\mathrm{Si} 5$ & $0.0287(2)$ & $0.0228(2)$ & $0.0295(2)$ & $-0.00127(18)$ & -0.00988 (19) & $-0.00119(18)$ \\
\hline C111 & 0.0288 & $0.0663(4)$ & $0.0855(5)$ & 0.0061 & $-0.0147(3)$ & $0.0272(4)$ \\
\hline $\mathrm{Cl12}$ & $0.0766(5)$ & 0.0475 & $0.0344(3)$ & $0.0162(3)$ & $0.0127(3)$ & $-0.0050(2)$ \\
\hline $\mathrm{C} 121$ & $0.0423(3)$ & $0.0669(4)$ & 0.0530 & $-0.0301(3)$ & $-0.0132(3)$ & 0.0083 \\
\hline $\mathrm{Cl} 22$ & $0.0427(3)$ & $0.0432(3)$ & $0.0342(2)$ & $-0.0043(2)$ & $-0.0008(2)$ & $0.0144(2)$ \\
\hline $\mathrm{Cl} 31$ & $0.0582(3)$ & $0.0344(2)$ & 0.0360 & $-0.0095(2)$ & $-0.0090(2)$ & $-0.0121(2)$ \\
\hline $\mathrm{C} 132$ & $0.0410(3)$ & $0.0302(2)$ & $0.0445(3)$ & 0.00368 (19) & $-0.0119(2)$ & 0.00648 (19) \\
\hline $\mathrm{Cl} 41$ & $0.0336(2)$ & $0.0416(3)$ & $0.0253(2)$ & $-0.01096(19)$ & $-0.00306(17)$ & $0.00253(17)$ \\
\hline $\mathrm{Cl} 42$ & $0.0285(2)$ & $0.0429(3)$ & $0.0433(3)$ & $-0.00619(18)$ & -0.01663 (19) & $0.0021(2)$ \\
\hline $\mathrm{C} 151$ & $0.0404(3)$ & $0.0343(2)$ & $0.0516(3)$ & $-0.0089(2)$ & $-0.0198(2)$ & $0.0174(2)$ \\
\hline $\mathrm{C} 152$ & $0.0629(4)$ & $0.0337(3)$ & $0.0529(3)$ & $-0.0081(2)$ & -0.0251 & $-0.0133(2)$ \\
\hline N1 & $0.0310(8)$ & $0.0304(8)$ & $0.0275(7)$ & $-0.0052(6)$ & $-0.0080(6)$ & $0.0030(6)$ \\
\hline $\mathrm{C} 1$ & $0.0746(19)$ & $0.0423(13)$ & $0.0395(12)$ & $-0.0160(13)$ & $-0.0016(12)$ & $0.0076(10)$ \\
\hline $\mathrm{C} 2$ & $0.085(2)$ & $0.0343(12)$ & $0.0522(15)$ & $-0.0151(13)$ & $-0.0287(15)$ & $0.0117(10)$ \\
\hline $\mathrm{C} 3$ & $0.0530(14)$ & $0.0456(12)$ & $0.0365(11)$ & $-0.0105(10)$ & $-0.0187(10)$ & $-0.0010(9)$ \\
\hline $\mathrm{C} 4$ & $0.069(2)$ & $0.0598(18)$ & $0.087(2)$ & $-0.0171(15)$ & $-0.0524(19)$ & $-0.0019(16)$ \\
\hline $\mathrm{C} 5$ & $0.0357(11)$ & $0.0378(12)$ & $0.0634(16)$ & $-0.0041(9)$ & $-0.0110(11)$ & $0.0002(11)$ \\
\hline C6 & $0.0512(15)$ & 0.0407 (13) & $0.0554(15)$ & $-0.0079(11)$ & $0.0070(12)$ & $-0.0096(11)$ \\
\hline $\mathrm{C} 7$ & $0.0378(11)$ & $0.0392(11)$ & $0.0498(13)$ & $-0.0028(9)$ & $-0.0208(10)$ & $0.0006(9)$ \\
\hline $\mathrm{C} 8$ & $0.0378(12)$ & $0.0480(14)$ & $0.0643(17)$ & $-0.0150(10)$ & $-0.0042(11)$ & $-0.0017(12)$ \\
\hline N2 & $0.0230(9)$ & $0.0267(10)$ & $0.0268(10)$ & $-0.0036(8)$ & $-0.0063(8)$ & $0.0051(8)$ \\
\hline $\mathrm{C} 21$ & $0.0329(18)$ & $0.0228(16)$ & $0.040(2)$ & $-0.0042(14)$ & $-0.0058(16)$ & $0.0055(14)$ \\
\hline $\mathrm{C} 21^{\prime}$ & 0.0330 (19) & $0.043(2)$ & $0.0293(17)$ & $-0.0049(16)$ & $-0.0108(15)$ & $0.0050(15)$ \\
\hline $\mathrm{C} 22$ & $0.0471(15)$ & $0.073(2)$ & 0.0663 (19) & $-0.0141(14)$ & $-0.0078(13)$ & $-0.0342(16)$ \\
\hline $\mathrm{C} 23$ & $0.0252(16)$ & $0.0297(17)$ & $0.0327(17)$ & $-0.0030(13)$ & $-0.0095(14)$ & $0.0056(14)$ \\
\hline C23' & $0.0275(17)$ & $0.0336(18)$ & $0.0322(18)$ & $-0.0082(14)$ & $-0.0031(14)$ & 0.0067 (14) \\
\hline $\mathrm{C} 24$ & $0.0240(9)$ & $0.0589(16)$ & $0.0671(17)$ & $-0.0074(10)$ & $0.0005(10)$ & $-0.0163(13)$ \\
\hline N3 & $0.0243(10)$ & $0.0345(12)$ & $0.0329(11)$ & $0.0000(9)$ & $-0.0037(9)$ & $-0.0037(9)$ \\
\hline C31 & $0.072(4)$ & $0.037(2)$ & $0.040(2)$ & $-0.006(2)$ & $-0.009(2)$ & -0.0055 (19) \\
\hline $\mathrm{C} 31^{\prime}$ & $0.030(2)$ & $0.081(4)$ & $0.043(3)$ & $-0.015(2)$ & $-0.0107(18)$ & $0.013(2)$ \\
\hline $\mathrm{C} 32$ & $0.146(4)$ & $0.103(3)$ & $0.080(3)$ & $-0.084(3)$ & $-0.061(3)$ & $0.023(2)$ \\
\hline $\mathrm{C} 33$ & $0.034(2)$ & $0.049(2)$ & $0.0292(18)$ & $0.0039(18)$ & $0.0004(16)$ & $-0.0031(17)$ \\
\hline C33' & $0.033(2)$ & $0.055(3)$ & $0.045(2)$ & 0.0018 (19) & $-0.0118(19)$ & 0.009 (2) \\
\hline $\mathrm{C} 34$ & $0.083(2)$ & $0.110(3)$ & $0.0381(14)$ & $-0.048(2)$ & $-0.0207(15)$ & $0.0216(16)$ \\
\hline C1L & $0.0620(17)$ & $0.0570(16)$ & 0.0647 (18) & $-0.0176(14)$ & $-0.0369(15)$ & $0.0150(13)$ \\
\hline Cl1A & 0.0500 & $0.0452(3)$ & $0.0797(5)$ & $-0.0104(3)$ & -0.0265 & 0.0018 \\
\hline Cl1B & $0.0613(4)$ & $0.0527(4)$ & $0.0685(5)$ & -0.0195 & $-0.0237(4)$ & $0.0092(3)$ \\
\hline $\mathrm{C} 2 \mathrm{~L}$ & 0.0639 (19) & $0.078(2)$ & $0.0503(16)$ & $0.0043(16)$ & $-0.0266(14)$ & $-0.0118(15)$ \\
\hline $\mathrm{C} 12 \mathrm{~A}$ & $0.0746(5)$ & $0.0622(4)$ & 0.0588 & $0.0015(4)$ & $-0.0367(4)$ & -0.0093 \\
\hline
\end{tabular}




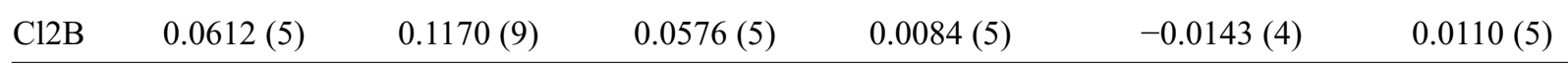

Geometric parameters $(\AA, \stackrel{o}{)}$

\begin{tabular}{|c|c|c|c|}
\hline $\mathrm{Si} 1-\mathrm{Cl12}$ & $2.0805(9)$ & $\mathrm{C} 22-\mathrm{H} 22 \mathrm{~B}$ & 0.9800 \\
\hline $\mathrm{Si} 1-\mathrm{C} 111$ & $2.0906(9)$ & $\mathrm{C} 22-\mathrm{H} 22 \mathrm{C}$ & 0.9800 \\
\hline $\mathrm{Si} 1-\mathrm{Si} 2$ & $2.3386(8)$ & $\mathrm{C} 22-\mathrm{H} 22 \mathrm{D}$ & 0.9800 \\
\hline $\mathrm{Si} 1-\mathrm{Si} 5$ & $2.3469(8)$ & $\mathrm{C} 22-\mathrm{H} 22 \mathrm{E}$ & 0.9800 \\
\hline $\mathrm{Si} 2-\mathrm{C} 121$ & $2.0910(8)$ & $\mathrm{C} 22-\mathrm{H} 22 \mathrm{~F}$ & 0.9800 \\
\hline $\mathrm{Si} 2-\mathrm{Cl} 22$ & $2.1102(8)$ & $\mathrm{C} 23-\mathrm{C} 24$ & $1.604(5)$ \\
\hline $\mathrm{Si} 2-\mathrm{Si} 3$ & $2.3465(7)$ & $\mathrm{C} 23-\mathrm{H} 23 \mathrm{~A}$ & 0.9900 \\
\hline $\mathrm{Si} 3-\mathrm{Cl} 31$ & $2.0849(7)$ & $\mathrm{C} 23-\mathrm{H} 23 \mathrm{~B}$ & 0.9900 \\
\hline $\mathrm{Si} 3-\mathrm{Cl} 32$ & $2.0967(8)$ & $\mathrm{C} 23^{\prime}-\mathrm{C} 24$ & $1.622(5)$ \\
\hline $\mathrm{Si} 3-\mathrm{Si} 4$ & $2.3419(7)$ & $\mathrm{C} 23^{\prime}-\mathrm{H} 23 \mathrm{C}$ & 0.9900 \\
\hline $\mathrm{Si} 4-\mathrm{Cl} 41$ & $2.0977(7)$ & $\mathrm{C} 23^{\prime}-\mathrm{H} 23 \mathrm{D}$ & 0.9900 \\
\hline $\mathrm{Si} 4-\mathrm{Cl} 42$ & $2.0993(7)$ & $\mathrm{C} 24-\mathrm{H} 24 \mathrm{~A}$ & 0.9800 \\
\hline $\mathrm{Si} 4-\mathrm{Si} 5$ & $2.3473(7)$ & $\mathrm{C} 24-\mathrm{H} 24 \mathrm{~B}$ & 0.9800 \\
\hline $\mathrm{Si} 5-\mathrm{Cl} 51$ & $2.0814(7)$ & $\mathrm{C} 24-\mathrm{H} 24 \mathrm{C}$ & 0.9800 \\
\hline $\mathrm{Si} 5-\mathrm{Cl} 52$ & $2.0890(8)$ & $\mathrm{C} 24-\mathrm{H} 24 \mathrm{D}$ & 0.9800 \\
\hline $\mathrm{N} 1-\mathrm{C} 3$ & $1.510(3)$ & $\mathrm{C} 24-\mathrm{H} 24 \mathrm{E}$ & 0.9800 \\
\hline $\mathrm{N} 1-\mathrm{C} 1$ & $1.516(3)$ & $\mathrm{C} 24-\mathrm{H} 24 \mathrm{~F}$ & 0.9800 \\
\hline $\mathrm{N} 1-\mathrm{C} 7$ & $1.519(3)$ & $\mathrm{N} 3-\mathrm{C} 31^{\mathrm{ii}}$ & $1.487(5)$ \\
\hline $\mathrm{N} 1-\mathrm{C} 5$ & $1.533(3)$ & $\mathrm{N} 3-\mathrm{C} 31$ & $1.487(5)$ \\
\hline $\mathrm{C} 1-\mathrm{C} 2$ & $1.526(4)$ & $\mathrm{N} 3-\mathrm{C} 33^{\mathrm{ii}}$ & $1.491(4)$ \\
\hline $\mathrm{C} 1-\mathrm{H} 1 \mathrm{~A}$ & 0.9900 & $\mathrm{~N} 3-\mathrm{C} 33$ & $1.491(4)$ \\
\hline $\mathrm{C} 1-\mathrm{H} 1 \mathrm{~B}$ & 0.9900 & $\mathrm{~N} 3-\mathrm{C} 31^{\prime \text { ii }}$ & $1.530(5)$ \\
\hline $\mathrm{C} 2-\mathrm{H} 2 \mathrm{~A}$ & 0.9800 & $\mathrm{~N} 3-\mathrm{C} 31^{\prime}$ & $1.530(5)$ \\
\hline $\mathrm{C} 2-\mathrm{H} 2 \mathrm{~B}$ & 0.9800 & $\mathrm{~N} 3-\mathrm{C} 33^{\prime \text { ii }}$ & $1.561(5)$ \\
\hline $\mathrm{C} 2-\mathrm{H} 2 \mathrm{C}$ & 0.9800 & N3-C33' & $1.561(5)$ \\
\hline $\mathrm{C} 3-\mathrm{C} 4$ & $1.511(4)$ & $\mathrm{C} 31-\mathrm{C} 32$ & $1.625(8)$ \\
\hline $\mathrm{C} 3-\mathrm{H} 3 \mathrm{~A}$ & 0.9900 & $\mathrm{C} 31-\mathrm{H} 31 \mathrm{~A}$ & 0.9900 \\
\hline C $3-\mathrm{H} 3 \mathrm{~B}$ & 0.9900 & С $31-\mathrm{H} 31 \mathrm{~B}$ & 0.9900 \\
\hline $\mathrm{C} 4-\mathrm{H} 4 \mathrm{~A}$ & 0.9800 & $\mathrm{C} 31^{\prime}-\mathrm{C} 32$ & $1.579(8)$ \\
\hline $\mathrm{C} 4-\mathrm{H} 4 \mathrm{~B}$ & 0.9800 & $\mathrm{C} 31^{\prime}-\mathrm{H} 31 \mathrm{C}$ & 0.9900 \\
\hline $\mathrm{C} 4-\mathrm{H} 4 \mathrm{C}$ & 0.9800 & $\mathrm{C} 31^{\prime}-\mathrm{H} 31 \mathrm{D}$ & 0.9900 \\
\hline $\mathrm{C} 5-\mathrm{C} 6$ & $1.514(4)$ & $\mathrm{C} 32-\mathrm{H} 32 \mathrm{~A}$ & 0.9800 \\
\hline C5-H5A & 0.9900 & С $32-\mathrm{H} 32 \mathrm{~B}$ & 0.9800 \\
\hline C5-H5B & 0.9900 & $\mathrm{C} 32-\mathrm{H} 32 \mathrm{C}$ & 0.9800 \\
\hline C6-H6A & 0.9800 & C32-H32D & 0.9800 \\
\hline $\mathrm{C} 6-\mathrm{H} 6 \mathrm{~B}$ & 0.9800 & C32-H32E & 0.9800 \\
\hline $\mathrm{C} 6-\mathrm{H} 6 \mathrm{C}$ & 0.9800 & $\mathrm{C} 32-\mathrm{H} 32 \mathrm{~F}$ & 0.9800 \\
\hline $\mathrm{C} 7-\mathrm{C} 8$ & $1.495(4)$ & $\mathrm{C} 33-\mathrm{C} 34$ & $1.656(6)$ \\
\hline C7-H7A & 0.9900 & C33-H33A & 0.9900 \\
\hline C7-H7B & 0.9900 & С $33-\mathrm{H} 33 \mathrm{~B}$ & 0.9900 \\
\hline $\mathrm{C} 8-\mathrm{H} 8 \mathrm{~A}$ & 0.9800 & $\mathrm{C} 33^{\prime}-\mathrm{C} 34$ & $1.599(6)$ \\
\hline C $8-\mathrm{H} 8 \mathrm{~B}$ & 0.9800 & $\mathrm{C} 33^{\prime}-\mathrm{H} 33 \mathrm{C}$ & 0.9900 \\
\hline $\mathrm{C} 8-\mathrm{H} 8 \mathrm{C}$ & 0.9800 & $\mathrm{C} 33^{\prime}-\mathrm{H} 33 \mathrm{D}$ & 0.9900 \\
\hline $\mathrm{N} 2-\mathrm{C} 23^{\prime \mathrm{i}}$ & $1.508(4)$ & $\mathrm{C} 34-\mathrm{H} 34 \mathrm{~A}$ & 0.9800 \\
\hline
\end{tabular}




\begin{tabular}{|c|c|c|c|}
\hline $\mathrm{N} 2-\mathrm{C} 23^{\prime}$ & $1.508(4)$ & $\mathrm{C} 34-\mathrm{H} 34 \mathrm{~B}$ & 0.9800 \\
\hline $\mathrm{N} 2-\mathrm{C} 23^{\mathrm{i}}$ & $1.514(4)$ & $\mathrm{C} 34-\mathrm{H} 34 \mathrm{C}$ & 0.9800 \\
\hline $\mathrm{N} 2-\mathrm{C} 23$ & $1.514(4)$ & $\mathrm{C} 34-\mathrm{H} 34 \mathrm{D}$ & 0.9800 \\
\hline $\mathrm{N} 2-\mathrm{C} 21^{\prime \mathrm{i}}$ & $1.517(4)$ & $\mathrm{C} 34-\mathrm{H} 34 \mathrm{E}$ & 0.9800 \\
\hline $\mathrm{N} 2-\mathrm{C} 21^{\prime}$ & $1.517(4)$ & $\mathrm{C} 34-\mathrm{H} 34 \mathrm{~F}$ & 0.9800 \\
\hline $\mathrm{N} 2-\mathrm{C} 21$ & $1.533(4)$ & $\mathrm{C} 1 \mathrm{~L}-\mathrm{C} 11 \mathrm{~A}$ & $1.765(3)$ \\
\hline $\mathrm{N} 2-\mathrm{C} 21^{\mathrm{i}}$ & $1.533(4)$ & $\mathrm{C} 1 \mathrm{~L}-\mathrm{C} 11 \mathrm{~B}$ & $1.769(3)$ \\
\hline $\mathrm{C} 21-\mathrm{C} 22$ & $1.633(6)$ & $\mathrm{C} 1 \mathrm{~L}-\mathrm{H} 1 \mathrm{~L} 1$ & 0.9900 \\
\hline $\mathrm{C} 21-\mathrm{H} 21 \mathrm{~A}$ & 0.9900 & $\mathrm{C} 1 \mathrm{~L}-\mathrm{H} 1 \mathrm{~L} 2$ & 0.9900 \\
\hline $\mathrm{C} 21-\mathrm{H} 21 \mathrm{~B}$ & 0.9900 & $\mathrm{C} 2 \mathrm{~L}-\mathrm{Cl} 2 \mathrm{~B}$ & $1.746(4)$ \\
\hline $\mathrm{C} 21^{\prime}-\mathrm{C} 22$ & $1.636(6)$ & $\mathrm{C} 2 \mathrm{~L}-\mathrm{Cl} 2 \mathrm{~A}$ & $1.757(3)$ \\
\hline $\mathrm{C} 21^{\prime}-\mathrm{H} 21 \mathrm{C}$ & 0.9900 & $\mathrm{C} 2 \mathrm{~L}-\mathrm{H} 2 \mathrm{~L} 1$ & 0.9900 \\
\hline $\mathrm{C} 21^{\prime}-\mathrm{H} 21 \mathrm{D}$ & 0.9900 & $\mathrm{C} 2 \mathrm{~L}-\mathrm{H} 2 \mathrm{~L} 2$ & 0.9900 \\
\hline $\mathrm{C} 22-\mathrm{H} 22 \mathrm{~A}$ & 0.9800 & & \\
\hline $\mathrm{Cl12}-\mathrm{Si1}-\mathrm{Cl11}$ & $98.50(4)$ & $\mathrm{C} 21-\mathrm{C} 22-\mathrm{H} 22 \mathrm{~A}$ & 109.5 \\
\hline $\mathrm{C} 112-\mathrm{Si} 1-\mathrm{Si} 2$ & $113.28(3)$ & $\mathrm{C} 21-\mathrm{C} 22-\mathrm{H} 22 \mathrm{~B}$ & 109.5 \\
\hline $\mathrm{C} 111-\mathrm{Si} 1-\mathrm{Si} 2$ & $111.32(4)$ & $\mathrm{H} 22 \mathrm{~A}-\mathrm{C} 22-\mathrm{H} 22 \mathrm{~B}$ & 109.5 \\
\hline $\mathrm{Cl} 12-\mathrm{Si} 1-\mathrm{Si} 5$ & $111.49(4)$ & $\mathrm{C} 21-\mathrm{C} 22-\mathrm{H} 22 \mathrm{C}$ & 109.5 \\
\hline Cl11-Si1-Si5 & $113.90(4)$ & $\mathrm{H} 22 \mathrm{~A}-\mathrm{C} 22-\mathrm{H} 22 \mathrm{C}$ & 109.5 \\
\hline $\mathrm{Si} 2-\mathrm{Si} 1-\mathrm{Si} 5$ & $108.23(3)$ & $\mathrm{H} 22 \mathrm{~B}-\mathrm{C} 22-\mathrm{H} 22 \mathrm{C}$ & 109.5 \\
\hline $\mathrm{C} 121-\mathrm{Si} 2-\mathrm{Cl} 22$ & $98.77(4)$ & $\mathrm{C} 21^{\prime}-\mathrm{C} 22-\mathrm{H} 22 \mathrm{D}$ & 109.5 \\
\hline $\mathrm{C} 121-\mathrm{Si} 2-\mathrm{Si} 1$ & $112.27(4)$ & $\mathrm{C} 21^{\prime}-\mathrm{C} 22-\mathrm{H} 22 \mathrm{E}$ & 109.5 \\
\hline $\mathrm{Cl} 22-\mathrm{Si} 2-\mathrm{Si} 1$ & $111.72(3)$ & $\mathrm{H} 22 \mathrm{D}-\mathrm{C} 22-\mathrm{H} 22 \mathrm{E}$ & 109.5 \\
\hline $\mathrm{Cl} 21-\mathrm{Si} 2-\mathrm{Si} 3$ & $112.69(3)$ & $\mathrm{C} 21^{\prime}-\mathrm{C} 22-\mathrm{H} 22 \mathrm{~F}$ & 109.5 \\
\hline $\mathrm{Cl} 22-\mathrm{Si} 2-\mathrm{Si} 3$ & $113.01(3)$ & $\mathrm{H} 22 \mathrm{D}-\mathrm{C} 22-\mathrm{H} 22 \mathrm{~F}$ & 109.5 \\
\hline $\mathrm{Si} 1-\mathrm{Si} 2-\mathrm{Si} 3$ & $108.26(3)$ & $\mathrm{H} 22 \mathrm{E}-\mathrm{C} 22-\mathrm{H} 22 \mathrm{~F}$ & 109.5 \\
\hline $\mathrm{Cl} 31-\mathrm{Si} 3-\mathrm{Cl} 32$ & $100.07(3)$ & $\mathrm{N} 2-\mathrm{C} 23-\mathrm{C} 24$ & $110.3(3)$ \\
\hline $\mathrm{Cl} 31-\mathrm{Si} 3-\mathrm{Si} 4$ & $111.69(3)$ & $\mathrm{N} 2-\mathrm{C} 23-\mathrm{H} 23 \mathrm{~A}$ & 109.6 \\
\hline $\mathrm{Cl} 32-\mathrm{Si} 3-\mathrm{Si} 4$ & $111.61(3)$ & $\mathrm{C} 24-\mathrm{C} 23-\mathrm{H} 23 \mathrm{~A}$ & 109.6 \\
\hline $\mathrm{Cl} 31-\mathrm{Si} 3-\mathrm{Si} 2$ & $114.12(3)$ & $\mathrm{N} 2-\mathrm{C} 23-\mathrm{H} 23 \mathrm{~B}$ & 109.6 \\
\hline $\mathrm{Cl} 32-\mathrm{Si} 3-\mathrm{Si} 2$ & $111.88(3)$ & $\mathrm{C} 24-\mathrm{C} 23-\mathrm{H} 23 \mathrm{~B}$ & 109.6 \\
\hline $\mathrm{Si} 4-\mathrm{Si} 3-\mathrm{Si} 2$ & $107.47(3)$ & $\mathrm{H} 23 \mathrm{~A}-\mathrm{C} 23-\mathrm{H} 23 \mathrm{~B}$ & 108.1 \\
\hline $\mathrm{Cl} 41-\mathrm{Si} 4-\mathrm{Cl} 42$ & $98.87(3)$ & $\mathrm{N} 2-\mathrm{C} 23^{\prime}-\mathrm{C} 24$ & $109.6(3)$ \\
\hline $\mathrm{Cl} 41-\mathrm{Si} 4-\mathrm{Si} 3$ & $111.22(3)$ & $\mathrm{N} 2-\mathrm{C} 23^{\prime}-\mathrm{H} 23 \mathrm{C}$ & 109.7 \\
\hline $\mathrm{Cl} 42-\mathrm{Si} 4-\mathrm{Si} 3$ & $112.22(3)$ & $\mathrm{C} 24-\mathrm{C} 23^{\prime}-\mathrm{H} 23 \mathrm{C}$ & 109.7 \\
\hline C141-Si4-Si5 & $112.75(3)$ & $\mathrm{N} 2-\mathrm{C} 23^{\prime}-\mathrm{H} 23 \mathrm{D}$ & 109.7 \\
\hline $\mathrm{Cl} 42-\mathrm{Si} 4-\mathrm{Si} 5$ & $113.01(3)$ & $\mathrm{C} 24-\mathrm{C} 23^{\prime}-\mathrm{H} 23 \mathrm{D}$ & 109.7 \\
\hline $\mathrm{Si} 3-\mathrm{Si} 4-\mathrm{Si} 5$ & $108.60(3)$ & $\mathrm{H} 23 \mathrm{C}-\mathrm{C} 23^{\prime}-\mathrm{H} 23 \mathrm{D}$ & 108.2 \\
\hline $\mathrm{Cl} 51-\mathrm{Si} 5-\mathrm{Cl} 52$ & $99.88(3)$ & $\mathrm{C} 23-\mathrm{C} 24-\mathrm{H} 24 \mathrm{~A}$ & 109.5 \\
\hline $\mathrm{Cl} 151-\mathrm{Si} 5-\mathrm{Si} 1$ & $113.02(3)$ & $\mathrm{C} 23-\mathrm{C} 24-\mathrm{H} 24 \mathrm{~B}$ & 109.5 \\
\hline $\mathrm{Cl} 52-\mathrm{Si} 5-\mathrm{Si} 1$ & $112.15(3)$ & $\mathrm{H} 24 \mathrm{~A}-\mathrm{C} 24-\mathrm{H} 24 \mathrm{~B}$ & 109.5 \\
\hline $\mathrm{Cl} 51-\mathrm{Si} 5-\mathrm{Si} 4$ & $114.27(3)$ & $\mathrm{C} 23-\mathrm{C} 24-\mathrm{H} 24 \mathrm{C}$ & 109.5 \\
\hline $\mathrm{Cl} 52-\mathrm{Si} 5-\mathrm{Si} 4$ & $110.11(3)$ & $\mathrm{H} 24 \mathrm{~A}-\mathrm{C} 24-\mathrm{H} 24 \mathrm{C}$ & 109.5 \\
\hline $\mathrm{Si} 1-\mathrm{Si} 5-\mathrm{Si} 4$ & $107.38(3)$ & $\mathrm{H} 24 \mathrm{~B}-\mathrm{C} 24-\mathrm{H} 24 \mathrm{C}$ & 109.5 \\
\hline $\mathrm{C} 3-\mathrm{N} 1-\mathrm{C} 1$ & $111.90(19)$ & $\mathrm{C} 23^{\prime}-\mathrm{C} 24-\mathrm{H} 24 \mathrm{D}$ & 109.5 \\
\hline $\mathrm{C} 3-\mathrm{N} 1-\mathrm{C} 7$ & $108.98(18)$ & $\mathrm{C} 23^{\prime}-\mathrm{C} 24-\mathrm{H} 24 \mathrm{E}$ & 109.5 \\
\hline $\mathrm{C} 1-\mathrm{N} 1-\mathrm{C} 7$ & $109.2(2)$ & $\mathrm{H} 24 \mathrm{D}-\mathrm{C} 24-\mathrm{H} 24 \mathrm{E}$ & 109.5 \\
\hline
\end{tabular}




\begin{tabular}{|c|c|c|c|}
\hline $\mathrm{C} 3-\mathrm{N} 1-\mathrm{C} 5$ & $108.79(19)$ & $\mathrm{C} 23^{\prime}-\mathrm{C} 24-\mathrm{H} 24 \mathrm{~F}$ & 109.5 \\
\hline $\mathrm{C} 1-\mathrm{N} 1-\mathrm{C} 5$ & $108.2(2)$ & $\mathrm{H} 24 \mathrm{D}-\mathrm{C} 24-\mathrm{H} 24 \mathrm{~F}$ & 109.5 \\
\hline $\mathrm{C} 7-\mathrm{N} 1-\mathrm{C} 5$ & $109.76(17)$ & $\mathrm{H} 24 \mathrm{E}-\mathrm{C} 24-\mathrm{H} 24 \mathrm{~F}$ & 109.5 \\
\hline $\mathrm{N} 1-\mathrm{C} 1-\mathrm{C} 2$ & $115.0(2)$ & 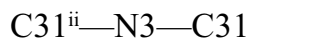 & $180.0(4)$ \\
\hline $\mathrm{N} 1-\mathrm{C} 1-\mathrm{H} 1 \mathrm{~A}$ & 108.5 & $\mathrm{C} 31^{\mathrm{ii}-\mathrm{N}} 3-\mathrm{C} 33^{\mathrm{ii}}$ & $66.3(3)$ \\
\hline $\mathrm{C} 2-\mathrm{C} 1-\mathrm{H} 1 \mathrm{~A}$ & 108.5 & $\mathrm{C} 31-\mathrm{N} 3-\mathrm{C} 33^{\mathrm{ii}}$ & $113.7(3)$ \\
\hline $\mathrm{N} 1-\mathrm{C} 1-\mathrm{H} 1 \mathrm{~B}$ & 108.5 & 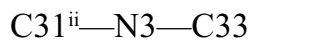 & $113.7(3)$ \\
\hline $\mathrm{C} 2-\mathrm{C} 1-\mathrm{H} 1 \mathrm{~B}$ & 108.5 & $\mathrm{C} 31-\mathrm{N} 3-\mathrm{C} 33$ & $66.3(3)$ \\
\hline $\mathrm{H} 1 \mathrm{~A}-\mathrm{C} 1-\mathrm{H} 1 \mathrm{~B}$ & 107.5 & $\mathrm{C} 33^{\mathrm{ii}}-\mathrm{N} 3-\mathrm{C} 33$ & 180.0 \\
\hline $\mathrm{C} 1-\mathrm{C} 2-\mathrm{H} 2 \mathrm{~A}$ & 109.5 & $\mathrm{C} 31^{\prime \mathrm{ii}}-\mathrm{N} 3-\mathrm{C} 31^{\prime}$ & $180.0(7)$ \\
\hline $\mathrm{C} 1-\mathrm{C} 2-\mathrm{H} 2 \mathrm{~B}$ & 109.5 & $\mathrm{C} 31^{\prime \text { ii }-N} 3-\mathrm{C} 33^{\prime \text { ii }}$ & $72.4(3)$ \\
\hline $\mathrm{H} 2 \mathrm{~A}-\mathrm{C} 2-\mathrm{H} 2 \mathrm{~B}$ & 109.5 & $\mathrm{C} 31^{\prime}-\mathrm{N} 3-\mathrm{C} 33^{\prime \text { ii }}$ & $107.6(3)$ \\
\hline $\mathrm{C} 1-\mathrm{C} 2-\mathrm{H} 2 \mathrm{C}$ & 109.5 & 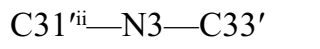 & $107.6(3)$ \\
\hline $\mathrm{H} 2 \mathrm{~A}-\mathrm{C} 2-\mathrm{H} 2 \mathrm{C}$ & 109.5 & $\mathrm{C} 31^{\prime}-\mathrm{N} 3-\mathrm{C} 33^{\prime}$ & $72.4(3)$ \\
\hline $\mathrm{H} 2 \mathrm{~B}-\mathrm{C} 2-\mathrm{H} 2 \mathrm{C}$ & 109.5 & 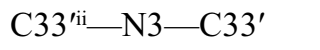 & $180.0(3)$ \\
\hline $\mathrm{N} 1-\mathrm{C} 3-\mathrm{C} 4$ & $115.5(2)$ & $\mathrm{N} 3-\mathrm{C} 31-\mathrm{C} 32$ & $110.4(4)$ \\
\hline $\mathrm{N} 1-\mathrm{C} 3-\mathrm{H} 3 \mathrm{~A}$ & 108.4 & $\mathrm{~N} 3-\mathrm{C} 31-\mathrm{H} 31 \mathrm{~A}$ & 109.6 \\
\hline $\mathrm{C} 4-\mathrm{C} 3-\mathrm{H} 3 \mathrm{~A}$ & 108.4 & $\mathrm{C} 32-\mathrm{C} 31-\mathrm{H} 31 \mathrm{~A}$ & 109.6 \\
\hline $\mathrm{N} 1-\mathrm{C} 3-\mathrm{H} 3 \mathrm{~B}$ & 108.4 & $\mathrm{~N} 3-\mathrm{C} 31-\mathrm{H} 31 \mathrm{~B}$ & 109.6 \\
\hline $\mathrm{C} 4-\mathrm{C} 3-\mathrm{H} 3 \mathrm{~B}$ & 108.4 & $\mathrm{C} 32-\mathrm{C} 31-\mathrm{H} 31 \mathrm{~B}$ & 109.6 \\
\hline $\mathrm{H} 3 \mathrm{~A}-\mathrm{C} 3-\mathrm{H} 3 \mathrm{~B}$ & 107.5 & $\mathrm{H} 31 \mathrm{~A}-\mathrm{C} 31-\mathrm{H} 31 \mathrm{~B}$ & 108.1 \\
\hline $\mathrm{C} 3-\mathrm{C} 4-\mathrm{H} 4 \mathrm{~A}$ & 109.5 & $\mathrm{~N} 3-\mathrm{C} 31^{\prime}-\mathrm{C} 32$ & $110.7(4)$ \\
\hline $\mathrm{C} 3-\mathrm{C} 4-\mathrm{H} 4 \mathrm{~B}$ & 109.5 & $\mathrm{~N} 3-\mathrm{C} 31^{\prime}-\mathrm{H} 31 \mathrm{C}$ & 109.5 \\
\hline $\mathrm{H} 4 \mathrm{~A}-\mathrm{C} 4-\mathrm{H} 4 \mathrm{~B}$ & 109.5 & $\mathrm{C} 32-\mathrm{C} 31^{\prime}-\mathrm{H} 31 \mathrm{C}$ & 109.5 \\
\hline $\mathrm{C} 3-\mathrm{C} 4-\mathrm{H} 4 \mathrm{C}$ & 109.5 & $\mathrm{~N} 3-\mathrm{C} 31^{\prime}-\mathrm{H} 31 \mathrm{D}$ & 109.5 \\
\hline $\mathrm{H} 4 \mathrm{~A}-\mathrm{C} 4-\mathrm{H} 4 \mathrm{C}$ & 109.5 & $\mathrm{C} 32-\mathrm{C} 31^{\prime}-\mathrm{H} 31 \mathrm{D}$ & 109.5 \\
\hline $\mathrm{H} 4 \mathrm{~B}-\mathrm{C} 4-\mathrm{H} 4 \mathrm{C}$ & 109.5 & $\mathrm{H} 31 \mathrm{C}-\mathrm{C} 31^{\prime}-\mathrm{H} 31 \mathrm{D}$ & 108.1 \\
\hline $\mathrm{C} 6-\mathrm{C} 5-\mathrm{N} 1$ & $115.2(2)$ & $\mathrm{C} 31-\mathrm{C} 32-\mathrm{H} 32 \mathrm{~A}$ & 109.5 \\
\hline $\mathrm{C} 6-\mathrm{C} 5-\mathrm{H} 5 \mathrm{~A}$ & 108.5 & $\mathrm{C} 31-\mathrm{C} 32-\mathrm{H} 32 \mathrm{~B}$ & 109.5 \\
\hline $\mathrm{N} 1-\mathrm{C} 5-\mathrm{H} 5 \mathrm{~A}$ & 108.5 & $\mathrm{H} 32 \mathrm{~A}-\mathrm{C} 32-\mathrm{H} 32 \mathrm{~B}$ & 109.5 \\
\hline $\mathrm{C} 6-\mathrm{C} 5-\mathrm{H} 5 \mathrm{~B}$ & 108.5 & $\mathrm{C} 31-\mathrm{C} 32-\mathrm{H} 32 \mathrm{C}$ & 109.5 \\
\hline $\mathrm{N} 1-\mathrm{C} 5-\mathrm{H} 5 \mathrm{~B}$ & 108.5 & $\mathrm{H} 32 \mathrm{~A}-\mathrm{C} 32-\mathrm{H} 32 \mathrm{C}$ & 109.5 \\
\hline $\mathrm{H} 5 \mathrm{~A}-\mathrm{C} 5-\mathrm{H} 5 \mathrm{~B}$ & 107.5 & $\mathrm{H} 32 \mathrm{~B}-\mathrm{C} 32-\mathrm{H} 32 \mathrm{C}$ & 109.5 \\
\hline $\mathrm{C} 5-\mathrm{C} 6-\mathrm{H} 6 \mathrm{~A}$ & 109.5 & $\mathrm{C} 31^{\prime}-\mathrm{C} 32-\mathrm{H} 32 \mathrm{D}$ & 109.5 \\
\hline $\mathrm{C} 5-\mathrm{C} 6-\mathrm{H} 6 \mathrm{~B}$ & 109.5 & $\mathrm{C} 31^{\prime}-\mathrm{C} 32-\mathrm{H} 32 \mathrm{E}$ & 109.5 \\
\hline $\mathrm{H} 6 \mathrm{~A}-\mathrm{C} 6-\mathrm{H} 6 \mathrm{~B}$ & 109.5 & $\mathrm{H} 32 \mathrm{D}-\mathrm{C} 32-\mathrm{H} 32 \mathrm{E}$ & 109.5 \\
\hline $\mathrm{C} 5-\mathrm{C} 6-\mathrm{H} 6 \mathrm{C}$ & 109.5 & $\mathrm{C} 31^{\prime}-\mathrm{C} 32-\mathrm{H} 32 \mathrm{~F}$ & 109.5 \\
\hline $\mathrm{H} 6 \mathrm{~A}-\mathrm{C} 6-\mathrm{H} 6 \mathrm{C}$ & 109.5 & $\mathrm{H} 32 \mathrm{D}-\mathrm{C} 32-\mathrm{H} 32 \mathrm{~F}$ & 109.5 \\
\hline $\mathrm{H} 6 \mathrm{~B}-\mathrm{C} 6-\mathrm{H} 6 \mathrm{C}$ & 109.5 & $\mathrm{H} 32 \mathrm{E}-\mathrm{C} 32-\mathrm{H} 32 \mathrm{~F}$ & 109.5 \\
\hline $\mathrm{C} 8-\mathrm{C} 7-\mathrm{N} 1$ & $116.3(2)$ & $\mathrm{N} 3-\mathrm{C} 33-\mathrm{C} 34$ & $108.3(3)$ \\
\hline $\mathrm{C} 8-\mathrm{C} 7-\mathrm{H} 7 \mathrm{~A}$ & 108.2 & N3-C $33-\mathrm{H} 33 \mathrm{~A}$ & 110.0 \\
\hline $\mathrm{N} 1-\mathrm{C} 7-\mathrm{H} 7 \mathrm{~A}$ & 108.2 & $\mathrm{C} 34-\mathrm{C} 33-\mathrm{H} 33 \mathrm{~A}$ & 110.0 \\
\hline $\mathrm{C} 8-\mathrm{C} 7-\mathrm{H} 7 \mathrm{~B}$ & 108.2 & $\mathrm{~N} 3-\mathrm{C} 33-\mathrm{H} 33 \mathrm{~B}$ & 110.0 \\
\hline $\mathrm{N} 1-\mathrm{C} 7-\mathrm{H} 7 \mathrm{~B}$ & 108.2 & C34-C33-H33B & 110.0 \\
\hline $\mathrm{H} 7 \mathrm{~A}-\mathrm{C} 7-\mathrm{H} 7 \mathrm{~B}$ & 107.4 & $\mathrm{H} 33 \mathrm{~A}-\mathrm{C} 33-\mathrm{H} 33 \mathrm{~B}$ & 108.4 \\
\hline $\mathrm{C} 7-\mathrm{C} 8-\mathrm{H} 8 \mathrm{~A}$ & 109.5 & $\mathrm{~N} 3-\mathrm{C} 33^{\prime}-\mathrm{C} 34$ & $107.8(3)$ \\
\hline $\mathrm{C} 7-\mathrm{C} 8-\mathrm{H} 8 \mathrm{~B}$ & 109.5 & $\mathrm{~N} 3-\mathrm{C} 33^{\prime}-\mathrm{H} 33 \mathrm{C}$ & 110.1 \\
\hline $\mathrm{H} 8 \mathrm{~A}-\mathrm{C} 8-\mathrm{H} 8 \mathrm{~B}$ & 109.5 & $\mathrm{C} 34-\mathrm{C} 33^{\prime}-\mathrm{H} 33 \mathrm{C}$ & 110.1 \\
\hline
\end{tabular}




\begin{tabular}{|c|c|c|c|}
\hline $\mathrm{C} 7-\mathrm{C} 8-\mathrm{H} 8 \mathrm{C}$ & 109.5 & $\mathrm{~N} 3-\mathrm{C} 33^{\prime}-\mathrm{H} 33 \mathrm{D}$ & 110.1 \\
\hline $\mathrm{H} 8 \mathrm{~A}-\mathrm{C} 8-\mathrm{H} 8 \mathrm{C}$ & 109.5 & $\mathrm{C} 34-\mathrm{C} 33^{\prime}-\mathrm{H} 33 \mathrm{D}$ & 110.1 \\
\hline $\mathrm{H} 8 \mathrm{~B}-\mathrm{C} 8-\mathrm{H} 8 \mathrm{C}$ & 109.5 & $\mathrm{H} 33 \mathrm{C}-\mathrm{C} 33^{\prime}-\mathrm{H} 33 \mathrm{D}$ & 108.5 \\
\hline $\mathrm{C} 23^{\prime \prime}-\mathrm{N} 2-\mathrm{C} 23^{\prime}$ & 180.0 & $\mathrm{C} 33-\mathrm{C} 34-\mathrm{H} 34 \mathrm{~A}$ & 109.5 \\
\hline $\mathrm{C} 23^{\mathrm{i}}-\mathrm{N} 2-\mathrm{C} 23$ & $180.0(3)$ & $\mathrm{C} 33-\mathrm{C} 34-\mathrm{H} 34 \mathrm{~B}$ & 109.5 \\
\hline $\mathrm{C} 23^{\prime \mathrm{i}}-\mathrm{N} 2-\mathrm{C} 21^{\prime \mathrm{i}}$ & $111.7(2)$ & $\mathrm{H} 34 \mathrm{~A}-\mathrm{C} 34-\mathrm{H} 34 \mathrm{~B}$ & 109.5 \\
\hline $\mathrm{C} 23^{\prime}-\mathrm{N} 2-\mathrm{C} 21^{\prime i}$ & $68.3(2)$ & $\mathrm{C} 33-\mathrm{C} 34-\mathrm{H} 34 \mathrm{C}$ & 109.5 \\
\hline $\mathrm{C} 23^{\prime \prime}-\mathrm{N} 2-\mathrm{C} 21^{\prime}$ & $68.3(2)$ & $\mathrm{H} 34 \mathrm{~A}-\mathrm{C} 34-\mathrm{H} 34 \mathrm{C}$ & 109.5 \\
\hline $\mathrm{C} 23^{\prime}-\mathrm{N} 2-\mathrm{C} 21^{\prime}$ & $111.7(2)$ & $\mathrm{H} 34 \mathrm{~B}-\mathrm{C} 34-\mathrm{H} 34 \mathrm{C}$ & 109.5 \\
\hline $\mathrm{C} 21^{\prime \prime}-\mathrm{N} 2-\mathrm{C} 21^{\prime}$ & 180.0 & $\mathrm{C} 33^{\prime}-\mathrm{C} 34-\mathrm{H} 34 \mathrm{D}$ & 109.5 \\
\hline $\mathrm{C} 23^{\mathrm{i}}-\mathrm{N} 2-\mathrm{C} 21$ & $69.5(2)$ & $\mathrm{C} 33^{\prime}-\mathrm{C} 34-\mathrm{H} 34 \mathrm{E}$ & 109.5 \\
\hline $\mathrm{C} 23-\mathrm{N} 2-\mathrm{C} 21$ & $110.5(2)$ & $\mathrm{H} 34 \mathrm{D}-\mathrm{C} 34-\mathrm{H} 34 \mathrm{E}$ & 109.5 \\
\hline $\mathrm{C} 23^{\mathrm{i}}-\mathrm{N} 2-\mathrm{C} 21^{\mathrm{i}}$ & $110.5(2)$ & $\mathrm{C} 33^{\prime}-\mathrm{C} 34-\mathrm{H} 34 \mathrm{~F}$ & 109.5 \\
\hline $\mathrm{C} 23-\mathrm{N} 2-\mathrm{C} 21^{\mathrm{i}}$ & $69.5(2)$ & $\mathrm{H} 34 \mathrm{D}-\mathrm{C} 34-\mathrm{H} 34 \mathrm{~F}$ & 109.5 \\
\hline $\mathrm{C} 21-\mathrm{N} 2-\mathrm{C} 21^{\mathrm{i}}$ & $180.0(4)$ & $\mathrm{H} 34 \mathrm{E}-\mathrm{C} 34-\mathrm{H} 34 \mathrm{~F}$ & 109.5 \\
\hline $\mathrm{N} 2-\mathrm{C} 21-\mathrm{C} 22$ & $107.8(3)$ & $\mathrm{C} 11 \mathrm{~A}-\mathrm{C} 1 \mathrm{~L}-\mathrm{C} 11 \mathrm{~B}$ & $110.64(16)$ \\
\hline $\mathrm{N} 2-\mathrm{C} 21-\mathrm{H} 21 \mathrm{~A}$ & 110.2 & $\mathrm{C} 11 \mathrm{~A}-\mathrm{C} 1 \mathrm{~L}-\mathrm{H} 1 \mathrm{~L} 1$ & 109.5 \\
\hline $\mathrm{C} 22-\mathrm{C} 21-\mathrm{H} 21 \mathrm{~A}$ & 110.2 & $\mathrm{C} 11 \mathrm{~B}-\mathrm{C} 1 \mathrm{~L}-\mathrm{H} 1 \mathrm{~L} 1$ & 109.5 \\
\hline $\mathrm{N} 2-\mathrm{C} 21-\mathrm{H} 21 \mathrm{~B}$ & 110.2 & $\mathrm{C} 11 \mathrm{~A}-\mathrm{C} 1 \mathrm{~L}-\mathrm{H} 1 \mathrm{~L} 2$ & 109.5 \\
\hline $\mathrm{C} 22-\mathrm{C} 21-\mathrm{H} 21 \mathrm{~B}$ & 110.2 & $\mathrm{Cl1B}-\mathrm{C} 1 \mathrm{~L}-\mathrm{H} 1 \mathrm{~L} 2$ & 109.5 \\
\hline $\mathrm{H} 21 \mathrm{~A}-\mathrm{C} 21-\mathrm{H} 21 \mathrm{~B}$ & 108.5 & $\mathrm{H} 1 \mathrm{~L} 1-\mathrm{C} 1 \mathrm{~L}-\mathrm{H} 1 \mathrm{~L} 2$ & 108.1 \\
\hline $\mathrm{N} 2-\mathrm{C} 21^{\prime}-\mathrm{C} 22$ & $108.4(3)$ & $\mathrm{Cl} 2 \mathrm{~B}-\mathrm{C} 2 \mathrm{~L}-\mathrm{Cl} 2 \mathrm{~A}$ & $111.70(18)$ \\
\hline $\mathrm{N} 2-\mathrm{C} 21^{\prime}-\mathrm{H} 21 \mathrm{C}$ & 110.0 & $\mathrm{Cl} 2 \mathrm{~B}-\mathrm{C} 2 \mathrm{~L}-\mathrm{H} 2 \mathrm{~L} 1$ & 109.3 \\
\hline $\mathrm{C} 22-\mathrm{C} 21^{\prime}-\mathrm{H} 21 \mathrm{C}$ & 110.0 & $\mathrm{Cl} 2 \mathrm{~A}-\mathrm{C} 2 \mathrm{~L}-\mathrm{H} 2 \mathrm{~L} 1$ & 109.3 \\
\hline $\mathrm{N} 2-\mathrm{C} 21^{\prime}-\mathrm{H} 21 \mathrm{D}$ & 110.0 & $\mathrm{Cl} 2 \mathrm{~B}-\mathrm{C} 2 \mathrm{~L}-\mathrm{H} 2 \mathrm{~L} 2$ & 109.3 \\
\hline $\mathrm{C} 22-\mathrm{C} 21^{\prime}-\mathrm{H} 21 \mathrm{D}$ & 110.0 & $\mathrm{Cl} 2 \mathrm{~A}-\mathrm{C} 2 \mathrm{~L}-\mathrm{H} 2 \mathrm{~L} 2$ & 109.3 \\
\hline $\mathrm{H} 21 \mathrm{C}-\mathrm{C} 21^{\prime}-\mathrm{H} 21 \mathrm{D}$ & 108.4 & $\mathrm{H} 2 \mathrm{~L} 1-\mathrm{C} 2 \mathrm{~L}-\mathrm{H} 2 \mathrm{~L} 2$ & 107.9 \\
\hline $\mathrm{C} 3-\mathrm{N} 1-\mathrm{C} 1-\mathrm{C} 2$ & $52.6(3)$ & $\mathrm{C} 23^{\prime}{ }^{\prime}-\mathrm{N} 2-\mathrm{C} 21^{\prime}-\mathrm{C} 22$ & $-117.4(3)$ \\
\hline $\mathrm{C} 7-\mathrm{N} 1-\mathrm{C} 1-\mathrm{C} 2$ & $-68.1(3)$ & $\mathrm{C} 23^{\prime}-\mathrm{N} 2-\mathrm{C} 21^{\prime}-\mathrm{C} 22$ & $62.6(3)$ \\
\hline $\mathrm{C} 5-\mathrm{N} 1-\mathrm{C} 1-\mathrm{C} 2$ & $172.4(3)$ & $\mathrm{C} 21-\mathrm{N} 2-\mathrm{C} 23-\mathrm{C} 24$ & $-62.3(3)$ \\
\hline $\mathrm{C} 1-\mathrm{N} 1-\mathrm{C} 3-\mathrm{C} 4$ & $53.9(3)$ & $\mathrm{C} 21^{\mathrm{i}}-\mathrm{N} 2-\mathrm{C} 23-\mathrm{C} 24$ & $117.7(3)$ \\
\hline $\mathrm{C} 7-\mathrm{N} 1-\mathrm{C} 3-\mathrm{C} 4$ & $174.8(2)$ & $\mathrm{C} 21^{\prime \mathrm{i}}-\mathrm{N} 2-\mathrm{C} 23^{\prime}-\mathrm{C} 24$ & $-119.9(3)$ \\
\hline $\mathrm{C} 5-\mathrm{N} 1-\mathrm{C} 3-\mathrm{C} 4$ & $-65.6(3)$ & $\mathrm{C} 21^{\prime}-\mathrm{N} 2-\mathrm{C} 23^{\prime}-\mathrm{C} 24$ & $60.1(3)$ \\
\hline $\mathrm{C} 3-\mathrm{N} 1-\mathrm{C} 5-\mathrm{C} 6$ & $-168.9(2)$ & $\mathrm{C} 33^{\mathrm{ii}}-\mathrm{N} 3-\mathrm{C} 31-\mathrm{C} 32$ & $-49.5(5)$ \\
\hline $\mathrm{C} 1-\mathrm{N} 1-\mathrm{C} 5-\mathrm{C} 6$ & $69.3(3)$ & $\mathrm{C} 33-\mathrm{N} 3-\mathrm{C} 31-\mathrm{C} 32$ & $130.5(5)$ \\
\hline $\mathrm{C} 7-\mathrm{N} 1-\mathrm{C} 5-\mathrm{C} 6$ & $-49.8(3)$ & $\mathrm{C} 33^{\prime \prime i}-\mathrm{N} 3-\mathrm{C} 31^{\prime}-\mathrm{C} 32$ & $57.8(4)$ \\
\hline $\mathrm{C} 3-\mathrm{N} 1-\mathrm{C} 7-\mathrm{C} 8$ & $61.8(3)$ & $\mathrm{C} 33^{\prime}-\mathrm{N} 3-\mathrm{C} 31^{\prime}-\mathrm{C} 32$ & $-122.2(4)$ \\
\hline $\mathrm{C} 1-\mathrm{N} 1-\mathrm{C} 7-\mathrm{C} 8$ & $-175.7(2)$ & $\mathrm{C} 31^{\mathrm{ii}}-\mathrm{N} 3-\mathrm{C} 33-\mathrm{C} 34$ & $60.0(5)$ \\
\hline $\mathrm{C} 5-\mathrm{N} 1-\mathrm{C} 7-\mathrm{C} 8$ & $-57.2(3)$ & $\mathrm{C} 31-\mathrm{N} 3-\mathrm{C} 33-\mathrm{C} 34$ & $-120.0(5)$ \\
\hline $\mathrm{C} 23^{\mathrm{i}}-\mathrm{N} 2-\mathrm{C} 21-\mathrm{C} 22$ & $118.2(3)$ & $\mathrm{C} 31^{\prime \prime i}-\mathrm{N} 3-\mathrm{C} 33^{\prime}-\mathrm{C} 34$ & $-58.6(5)$ \\
\hline $\mathrm{C} 23-\mathrm{N} 2-\mathrm{C} 21-\mathrm{C} 22$ & $-61.8(3)$ & $\mathrm{C} 31^{\prime}-\mathrm{N} 3-\mathrm{C} 33^{\prime}-\mathrm{C} 34$ & $121.4(5)$ \\
\hline
\end{tabular}

Symmetry codes: (i) $-x,-y+1,-z+1$; (ii) $-x,-y+2,-z+1$.

Hydrogen-bond geometry $\left(\AA,{ }^{\circ}\right)$

\begin{tabular}{lllll}
\hline$D-\mathrm{H} \cdots A$ & $D-\mathrm{H}$ & $\mathrm{H} \cdots A$ & $D \cdots A$ & $D-\mathrm{H} \cdots A$ \\
\hline $\mathrm{C} 1-\mathrm{H} 1 B \cdots \mathrm{Cl} 42$ & 0.99 & 2.99 & $3.829(3)$ & 144
\end{tabular}




$\begin{array}{lllll}\mathrm{C} 2-\mathrm{H} 2 C \cdots \mathrm{Cl} 2 & 0.98 & 2.95 & 3.753(3) & 139 \\ \mathrm{C} 3-\mathrm{H} 3 A \cdots \mathrm{Cl} 52^{\mathrm{iii}} & 0.99 & 2.79 & 3.643(3) & 144 \\ \mathrm{C} 3-\mathrm{H} 3 B^{\cdots} \cdots \mathrm{Cl} 2 B^{\mathrm{iv}} & 0.99 & 2.98 & 3.804(3) & 142 \\ \mathrm{C} 5-\mathrm{H} 5 B \cdots \mathrm{Cl} 22^{\mathrm{v}} & 0.99 & 2.89 & 3.850(3) & 165 \\ \mathrm{C} 6-\mathrm{H} 6 B^{\cdots}{ }^{\mathrm{C}} \mathrm{Cl} 21^{\mathrm{v}} & 0.98 & 2.86 & 3.630(3) & 136 \\ \mathrm{C} 7-\mathrm{H} 7 A \cdots \mathrm{Cl} 2 & 0.99 & 2.86 & 3.394(2) & 115 \\ \mathrm{C} 22-\mathrm{H} 22 C \cdots \mathrm{Cl} 51^{\mathrm{vi}} & 0.98 & 2.89 & 3.847(4) & 165 \\ \mathrm{C} 22-\mathrm{H} 22 E \cdots \mathrm{Cl} 41 & 0.98 & 2.90 & 3.859(3) & 165 \\ \mathrm{C} 23-\mathrm{H} 23 B \cdots \mathrm{Cl} 1^{\mathrm{vi}} & 0.99 & 2.98 & 3.465(4) & 111 \\ \mathrm{C} 23-\mathrm{H} 23 C \cdots \mathrm{Cl} 42 & 0.99 & 2.87 & 3.497(4) & 122 \\ \mathrm{C} 24-\mathrm{H} 24 C \cdots \mathrm{Cl} 41 & 0.98 & 2.84 & 3.793(3) & 164 \\ \mathrm{C} 24-\mathrm{H} 24 E \cdots \mathrm{Cl} 151^{\mathrm{vi}} & 0.98 & 2.81 & 3.771(3) & 165 \\ \mathrm{C} 24-\mathrm{H} 24 F \cdots \mathrm{Cl} 2 A^{\mathrm{vi}} & 0.98 & 2.92 & 3.778(3) & 147 \\ \mathrm{C} 31^{\prime}-\mathrm{H} 31 C \cdots \mathrm{Cl} 31 & 0.99 & 2.95 & 3.434(5) & 111 \\ \mathrm{C} 32-\mathrm{H} 32 F \cdots \mathrm{Cl} 21^{\mathrm{vii}} & 0.98 & 2.76 & 3.584(4) & 142 \\ \mathrm{C} 33-\mathrm{H} 33 A \cdots \mathrm{Cl} 32^{\mathrm{ii}} & 0.99 & 2.94 & 3.515(4) & 118 \\ \mathrm{C} 33^{\prime}-\mathrm{H} 33 D \cdots \mathrm{Cl} 41 & 0.99 & 2.98 & 3.630(5) & 124 \\ \mathrm{C} 34-\mathrm{H} 34 A \cdots \mathrm{Cl} 1 B^{\mathrm{i}} & 0.98 & 2.93 & 3.556(3) & 123 \\ \mathrm{C} 34-\mathrm{H} 34 C \cdots \mathrm{Cl} 2 B & 0.98 & 2.89 & 3.716(4) & 142 \\ \mathrm{C} 34-\mathrm{H} 34 A \cdots \mathrm{Cl} 1 B^{\mathrm{i}} & 0.98 & 2.93 & 3.556(3) & 123 \\ \mathrm{C} 1 L-\mathrm{H} 1 L 1 \cdots \mathrm{Cl} 12^{\mathrm{iii}} & 0.99 & 2.90 & 3.421(3) & 114 \\ \mathrm{C} 2 L-\mathrm{H} 2 L 2 \cdots \mathrm{Cl} 41 & 0.99 & 2.96 & 3.465(3) & 113\end{array}$

Symmetry codes: (i) $-x,-y+1,-z+1$; (ii) $-x,-y+2,-z+1$; (iii) $-x+1,-y+1,-z$; (iv) $x, y, z-1$; (v) $x-1, y, z$; (vi) $-x+1,-y+1,-z+1$; (vii) $-x+1,-y+2,-z+1$. 